\title{
Distributed network processes account for the majority of variance in localized visual category selectivity
}

Cocuzza, Carrisa V. ${ }^{1,2}$, Sanchez-Romero, Ruben. ${ }^{1}$, Ito, Takuya. ${ }^{3}$, Mill, Ravi D. ${ }^{1}$, Keane, Brian P. ${ }^{4}$, Cole, Michael W. ${ }^{1}$

${ }^{1}$ Center for Molecular and Behavioral Neuroscience, Rutgers University, Newark, NJ, 07102

${ }^{2}$ Behavioral and Neural Sciences PhD Program, Rutgers University, Newark, NJ, 07102

${ }^{3}$ Department of Psychiatry, Yale University School of Medicine, New Haven, CT, 06520

${ }^{4}$ Departments of Psychiatry, Neuroscience, and Brain \& Cognitive Science, University of Rochester Medical Center, Rochester, NY, 14642

\section{Abstract}

In seeking to understand processes fundamental to brain function, the debate between localized versus distributed processing has persisted for over a century. High category selectivity of some visual regions has provided evidence for localized (within-region) processing. However, given recent evidence that distributed connectivity patterns are predictive of localized task-evoked activations, we hypothesized that even highly localized category-selective brain activations are primarily determined by the connectivity-specified convergence of distributed brain processes. Consistent with this, we utilized fMRI data from $\mathrm{N}=352$ human participants to find that distributed activity flow processes (specified by each region's unique "connectivity fingerprint") locally converge on visual cortex regions to confer the majority contribution to visual category selective responses to bodies, faces, places, and tools. This highlights a prominent role for functional network organization in the generation of localized visual category selectivity and provides evidence for a distributed convergence account of localized functionality in the human brain.

\section{Significance Statement}

A fundamental debate in neuroscience has persisted for over a century with strong evidence on both sides: to what extent do localized versus distributed processes drive brain function? The existence of category-selective regions within the visual cortex provides some of the clearest evidence supporting localized processing, wherein specialized functions (e.g., category selectivity to faces) are primarily generated by within-region processes. This localized account has been countered by the identification of distributed visual cortex category selectivity in the absence of category-selective 
regions. Here we provide groundwork evidence for a theory of distributed convergence, demonstrating that localized visual-category-selective responses can be accurately generated via distributed activity flow processes. These predictions were generated via each category-selective region's unique connectivity fingerprint, demonstrating that the human brain's distributed network architecture is sufficient to generate category selectivity in visual cortex regions that were historically seen as evidence for a primarily local account.

\section{Introduction}

A long-standing question in cognitive neuroscience concerns the degree to which local versus distributed mechanisms underlie neural processes. Competing frameworks posit the neural operations driving functional organization along a theoretical continuum. At one end, specialized local computations determine neural processing (Kanwisher, 2010; Schalk et al., 2017), and at the other end, distributed, flexible patterns of activity underlie functioning (Op de Beeck et al., 2008; Mur et al., 2009). Most modern neuroscience assumes (explicitly or implicitly) theory somewhere between these two extremes (Borra et al., 2010), but the allocation of distributed versus local processing in the generation of neural responses remains unknown. Accordingly, there is an outstanding gap in knowledge regarding a fundamental aspect of neural processing that has held back progress in many areas of neuroscience, such as vision.

Extensive evidence for cortical regions exhibiting highly selective responses to visual categories such as bodies (Downing et al., 2001; Taylor et al., 2007), faces (Kanwisher and Yovel, 2006), places (Park and Chun, 2009), and tools (Peelen and Downing, 2005) strongly supports a localizationist view. For instance, high selectivity for face images in the fusiform face area (FFA) has been validated with human neuroimaging (Kanwisher, 2010), neural recordings in non-human primates (Tsao et al., 2008), human lesion studies (Corrow et al., 2016), and brain stimulation in humans (Schalk et al., 2017). Thus, to the extent that visual category processing generalizes to other functions, one might conclude that brain processing is highly localized. In parallel, however, an influential study demonstrated that visual categories could be decoded from distributed yet overlapping activity patterns in ventral temporal cortex (VTC) even after highly category selective regions (e.g., FFA) were excluded from the analysis (Haxby et al., 2001). Contemporaneous work proposed that a cortical region's functioning is largely determined by its connectivity fingerprint (not to be confused with individual difference "connectome fingerprinting"; Finn et al., 2015), or, a region's pattern of cortico-cortical connections that specify what information that region receives and conveys (Passingham et al., 2002). Thus, connectivity fingerprints may not be just a byproduct of a region's function, but a key determinant (Mars et al., 2018). Over recent decades, the functional 
relevance of brain network connectivity has been borne out by a growing body of network neuroscience (Sporns, 2014) studies assessing structural, functional, and effective interactions (Friston, 2011; Reid et al., 2019) during rest and task (Cole et al., 2021). Further, work by Osher et al. (2016) found that structural connectivity fingerprints can predict visual category responses in select VTC regions. However, because statistical associations were used to make those predictions (rather than a generative/mechanistic model), it remains unknown why connectivity is predictive of visual category selectivity. Based on our recent work demonstrating the importance of activity flow in generating neurocognitive processes (Cole et al., 2016; Cocuzza et al., 2022), we hypothesized that highly localized visual category selectivity in visual cortex regions are primarily determined by distributed activity flowing over each region's connectivity fingerprint. By testing a model of such generative network processes, the present study provides evidence that activity flowing over a distributed network architecture is key to accounting for why connectivity can predict local activity.

Putting our hypothesis in a larger theoretical perspective that takes into account results supporting both localized and distributed processing, here we develop a mechanistic framework that we refer to as the distributed convergence theory. We propose that distributed activity, selected by a given region's connectivity fingerprint, converges on that region, conferring it with locally specialized functioning (e.g., category selectivity). One likely purpose of this localized convergence (e.g., in the FFA) is to represent related information (e.g., faces) distributed throughout the brain in a common space for efficient competition between alternative activity patterns. In this account, FFA may facilitate face recognition via implementing a winner-take-all computation (via local inhibition; Pajani et al., 2017) between alternate faces represented in FFA, which is consistent with many computational models of local neural functions (O'Reilly, 2001). Importantly, rather than these local (e.g., recurrent activity or competition due to local inhibition) processes, we propose that the selective activity observed in functionally specialized regions is generated primarily by distributed activity flowing over each region's connectivity fingerprint. Importantly, our primary hypothesis suggests that distributed connectivity patterns play an active, generative role in localized region-level activity, and predicts that estimating activity flow over a region's unique connectivity fingerprint would account for the majority of that region's localized task-evoked selectivity.

Importantly, evidence that distributed processing mechanisms provide the dominant influence on visual category selective responses does not discount the importance of local computations, but rather provides the groundwork for a distributed convergence theory of how brain network interactions can be sufficient to generate such local computations. Activity flow mapping is well-suited to test these hypotheses (relative to, e.g., standard encoding models and artificial neural networks) because all parameters 
are based on empirical quantities, rather than abstractions (Kriegeskorte and Douglas, 2019; Ito et al., 2020a). The parameter of brain connectivity (a region's unique connectivity fingerprint) is key to characterizing the processes that converge to generate local responses.

We tested our hypothesis - an initial step toward validating the distributed convergence theory in the scope of visual category selectivity - utilizing human functional magnetic resonance imaging (fMRI) resting- and task-state timeseries from the Human Connectome Project (HCP) (Van Essen et al., 2013) young adult release. We focused on category-specific representations of bodies, faces, places, and tools embedded in a working memory task. These visual categories correspond to functionally specialized visual cortex regions that are highly replicated in the literature, such as the fusiform face area (FFA) and the parahippocampal place area (PPA). In sets (or complexes) of functional ROIs in the visual cortex, we quantified actual and activity-flowpredicted visual category selectivity and estimated the degree to which distributed processes contributed to this selectivity. We probed the differential contributions of large-scale functional networks to activity flow processes, and used substitution null models to corroborate that a given ROI's connectivity fingerprint significantly shaped its visual category selectivity. Demonstrating that region-level visual category selectivity can be generated by distributed, connectivity-based processes would be evidence against primarily local accounts of neural processing. Systematically testing visual category selectivity via activity flow mapping constitutes an important first step in refining a distributed convergence framework for how functionally selective responses exhibited by local regions of cortex emerge within a distributed, functionally-organized network architecture.

\section{Results}

\section{Response profiles are captured by activity flow processes}

Our preliminary hypothesis was that activity flow processes could predict responses in classically studied visual cortex regions. Firstly, we extended prior work (Cole et al., 2016; Ito et al., 2020a; Cocuzza et al., 2022) by implementing a resting-state functional connectivity (rsFC) method (Sanchez-Romero and Cole, 2021) that estimates a more causally plausible network (Fig. 1A-B), and by analyzing a large cohort $(\mathrm{N}=352)$ split into discovery and replication datasets. These updates serve to improve the inferential validity of activity flow mapping results (Reid et al., 2019). Secondly, we quantified response profiles (also termed population receptive fields) of functional complexes identified as integral to four visual categories (see Methods). A response profile (rows of 
Fig. 1D) was defined as the task-evoked activity levels across all 24 HCP conditions. Establishing response profiles allowed us to verify that each complex was responsive to their respective visual categories (outlined in black: Fig. 1D right), and verify that activity flow mapping (Fig. 1D left) was reliable across a variety of cognitive domains.
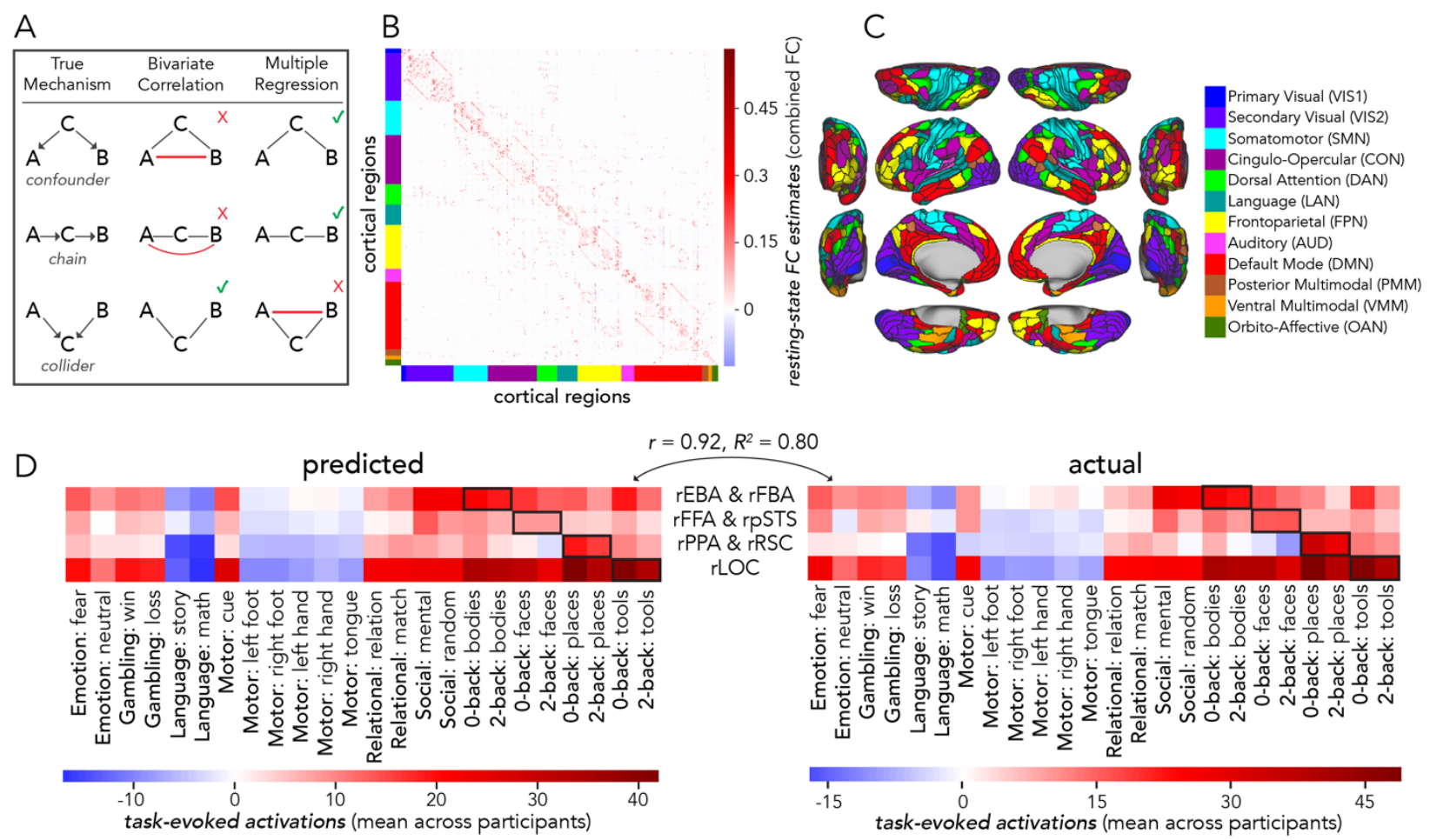

Figure 1. Predicted activations across all HCP conditions yielded response profiles for four functional complexes of interest. (A) Causally confounding graph patterns in standard FC estimation methods (adapted with permission from Sanchez-Romero and Cole, 2021). CombinedFC incorporates both bivariate and multiple regression measures such that confounders, chains, and colliders are accounted for. (B) The cross-participant ( $n=176$ ) average resting-state connectivity matrix (estimated via combinedFC) of 360 MMP parcels (Glasser et al., 2016), ordered along each axis per the Cole Anticevic brain-wide network partition (CAB-NP; Ji et al., 2019; color-coded on each axis to match panel C). This was the functional network organization utilized in the present study for activity flow mapping. (C) Cortical schematic of the CAB-NP and its 12 functional networks from Ji et al., 2019, reproduced with permission. (D) Response profiles (across all $24 \mathrm{HCP}$ conditions) of four complexes of interest to the present study (indicated along the $y$-axis; " $r$ " stands for right hemisphere); predicted versus actual (left and right respectively; mean across participants depicted in each panel). Black boxes highlight the $n$-back conditions that maintained visual semantic category embeddings germane to a given functional complex (e.g., 0-back bodies and 2-back bodies for the right EBA and right FBA). Activity flow mapping predicted response profiles with high accuracy suggesting that predicted response patterns of the functional complexes of interest were reliable across multiple cognitive domains.

Activity-flow-predicted response profiles maintained high prediction accuracy in both hemispheres (right: $r=0.92, \mathrm{MAE}=3.93, \mathrm{R}^{2}=0.80$; left: $r=0.92, \mathrm{MAE}=3.96, \mathrm{R}^{2}$ $=0.79$; replication: Table S1), which corroborates prior findings that activity flow 
processes (specified by connectivity patterns) can accurately predict task-evoked activity in held out brain regions across diverse cognitive domains (Cole et al., 2016). Moreover, this suggests that functional network organization (Fig. 1C) plays a prominent role in generating local response profiles. Subsequent analyses examined the processes involved in category-selective responses (i.e., focusing on black-outlined conditions in Fig. 1D), as conferred by activity flow mapping.

Local visual category selective responses are generated via distributed processing mechanisms

We next tested the hypothesis that the classically observed selectivity for visual categories in select visual cortex regions is shaped by their unique connectivity fingerprints. Each visual category studied enjoys an extensive literature reporting brain regions thought to represent (or compute) said categories and/or their component features (Methods Table 2). An emerging literature suggests that network interactions and/or distributed activation patterns are integral to visual category processing (Haxby et al., 2001; Norman-Haignere et al., 2012; Li et al., 2019). Here, we sought to test a core tenet of the distributed convergence theory by modeling activity flow processes (Cole et al., 2016). The four visual category models are reported in separate sections below with similar formatting to aid cross-comparison. The functional complexes identified based on prior literature (Fig. 2) were anatomically positioned along occipital (anterior and lateral to $\mathrm{V} 1$ ) and temporal (tending posterior and ventral to A1) cortices, which reflects the associative processing demands of visual category computations (Wierenga et al., 2009; Op de Beeck et al., 2019). Functional complex regions belonged primarily to the secondary visual (VIS2) network, with additional locations in dorsal attention (DAN), default mode (DMN), and language networks (LAN) (Fig. 2) (Ji et al., 2019). 

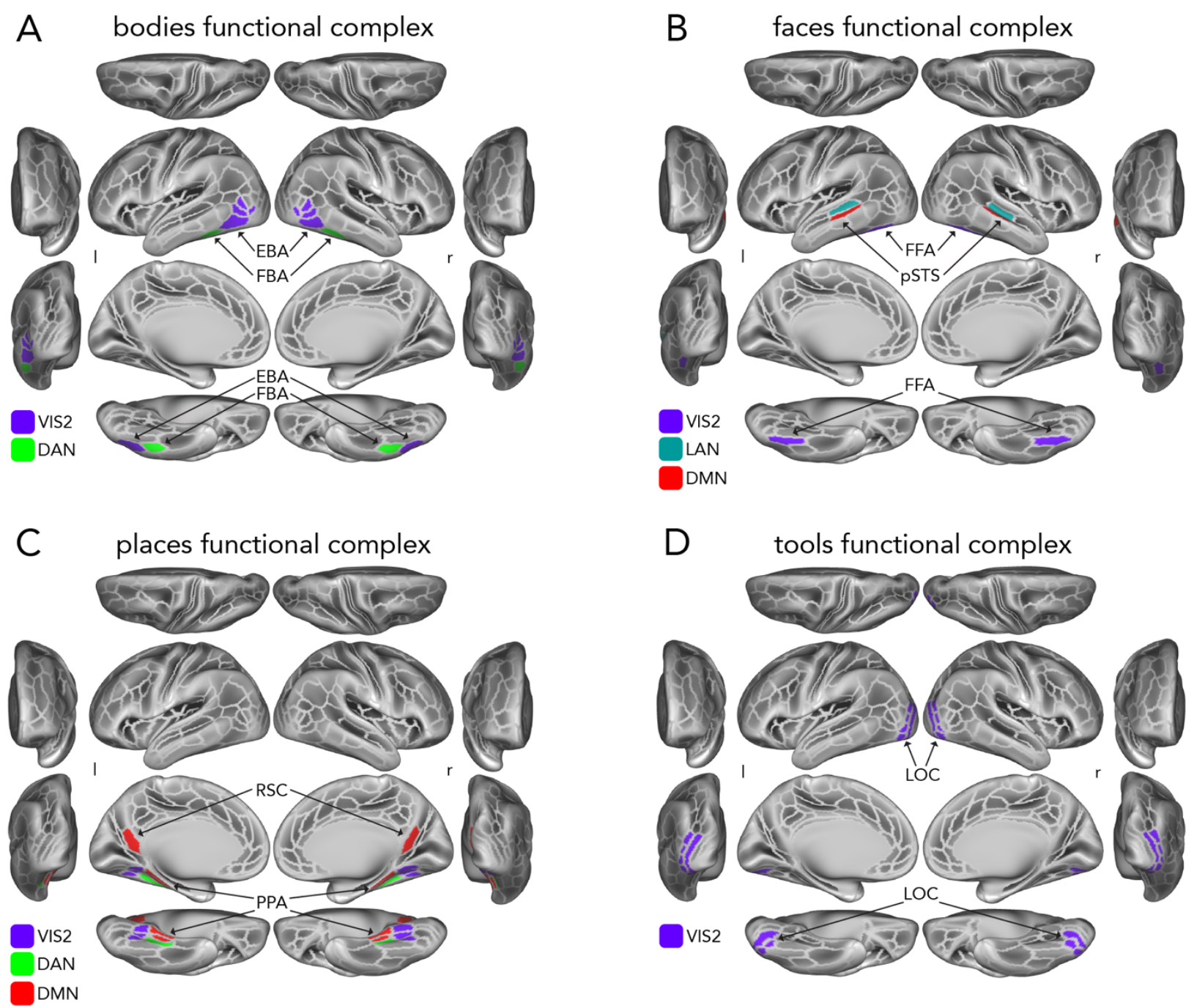

Figure 2. Visual categories and identified functional complexes. (B) A schematic of the cortical surface in three gross orientations, with MMP parcels (Glasser et al., 2016) outlined in silver and functional complexes relevant to processing body features (see Methods Tables 2-3) colored in based on CAB-NP network affiliations (Ji et al. 2019; Fig. 1C) and identified with text labels and arrows. EBA = extrastriate body area; FBA = fusiform body area. VIS2 = secondary visual network; DAN = dorsal attention network. " $r$ " denotes right hemisphere; "I" denotes left hemisphere. (B) Functional complexes related to face processing. FFA = fusiform body area; $\mathrm{PSTS}=$ posterior superior temporal sulcus; $\mathrm{LAN}=$ language network; DMN = default mode network. (C) Functional complexes related to places and scenes. RSC = retrosplenial complex; PPA = parahippocampal place area. (D) Functional complexes related to processing images of tools and objects. LOC = lateral occipital complex.

Our core hypothesis was that activity flow via the connectivity fingerprint of each functional complex (Fig. 2) is likely the primary determinant of their category-selective responses. Before this, we began with two confirmations essential for subsequent tests of this hypothesis. We first constrained prior activity flow mappings (Cole et al., 2016) to body, face, place, and tool conditions and compared actual versus activity-flowpredicted responses across the whole cortex (Fig. S1). We then focused on activations in 
each functional complex and in each hemisphere, and quantified "benchmark" contrasts to corroborate the literature by comparing the category of interest versus non-category responses (e.g., body versus non-body) in the actual and activity-flow-predicted data (note that category selectivity is reported subsequently). Whole-cortex activity-flowmapped responses to visual categories were highly accurate (bodies: $r=0.89$, MAE = 5.27, $R^{2}=0.78$; faces: $r=0.86, \mathrm{MAE}=5.83, \mathrm{R}^{2}=0.72 ;$ places: $r=0.88, \mathrm{MAE}=5.85, \mathrm{R}^{2}$ $=0.77$; tools: $r=0.89, \mathrm{MAE}=5.62, \mathrm{R}^{2}=0.78$; Fig. S1). Significant benchmark contrasts in each functional complex were also observed in both the actual (bodies: $t(175)=26.65$, $p=4.42 \times 10^{-63}$, Cohen's $d=1.42$, faces: $t(175)=20.37, p=5.26 \times 10^{-48}$, Cohen's $d=$ 1.23; places: $t(175)=39.53, p=2.95 \times 10^{-88}$, Cohen's $d=2.78$; tools: $t(175)=8.49, p=$ $4.71 \times 10^{-15}$, Cohen's $d=0.31$ ) and activity-flow-predicted data (bodies: $t(175)=17.91$, $p=1.79 \times 10^{-41}$, Cohen's $d=0.88$; faces: $t(175)=10.06, p=2.72 \times 10^{-19}$, Cohen's $d=$ 0.56; places: $t(175)=26.06 p=7.62 \times 10^{-62}$, Cohen's $d=1.78$; tools: $t(175)=9.94, p=$ $5.55 \times 10^{-19}$, Cohen's $d=0.32$ ) (Fig. S2) (left hemisphere and replication: Tables S1-S2).

Distributed processes contribute to body category selectivity

Processing human body images (Barch et al., 2013) is associated with the fusiform body area (FBA) and the extrastriate body area (EBA) (Downing et al., 2001; Taylor et al., 2007; Orgs et al., 2016), which are located in the lateral occipito-temporal cortex (Fig. 2A). EBA computations are thought to discriminate between bodily attributes and parts (Carey et al., 2019) and provide postural information to frontoparietal regions (Zimmermann et al., 2018), indicating a pivotal role in action planning. The FBA is thought to process images of whole bodies (Taylor et al., 2007) and pairs of body parts (Bratch et al., 2018). Extending our core hypothesis to the processing of body images, we hypothesized that activity flow via the connectivity fingerprint of the EBA and FBA (henceforth: EBA/FBA) (Fig. 3A-B) - its unique pattern of distributed cortical connections - is likely the primary determinant of its body-selective responses. 
bioRxiv preprint doi: https://doi.org/10.1101/2022.02.19.481103; this version posted February 22, 2022. The copyright holder for this preprint (which was not certified by peer review) is the author/funder, who has granted bioRxiv a license to display the preprint in perpetuity. It is made available under aCC-BY-NC-ND 4.0 International license.
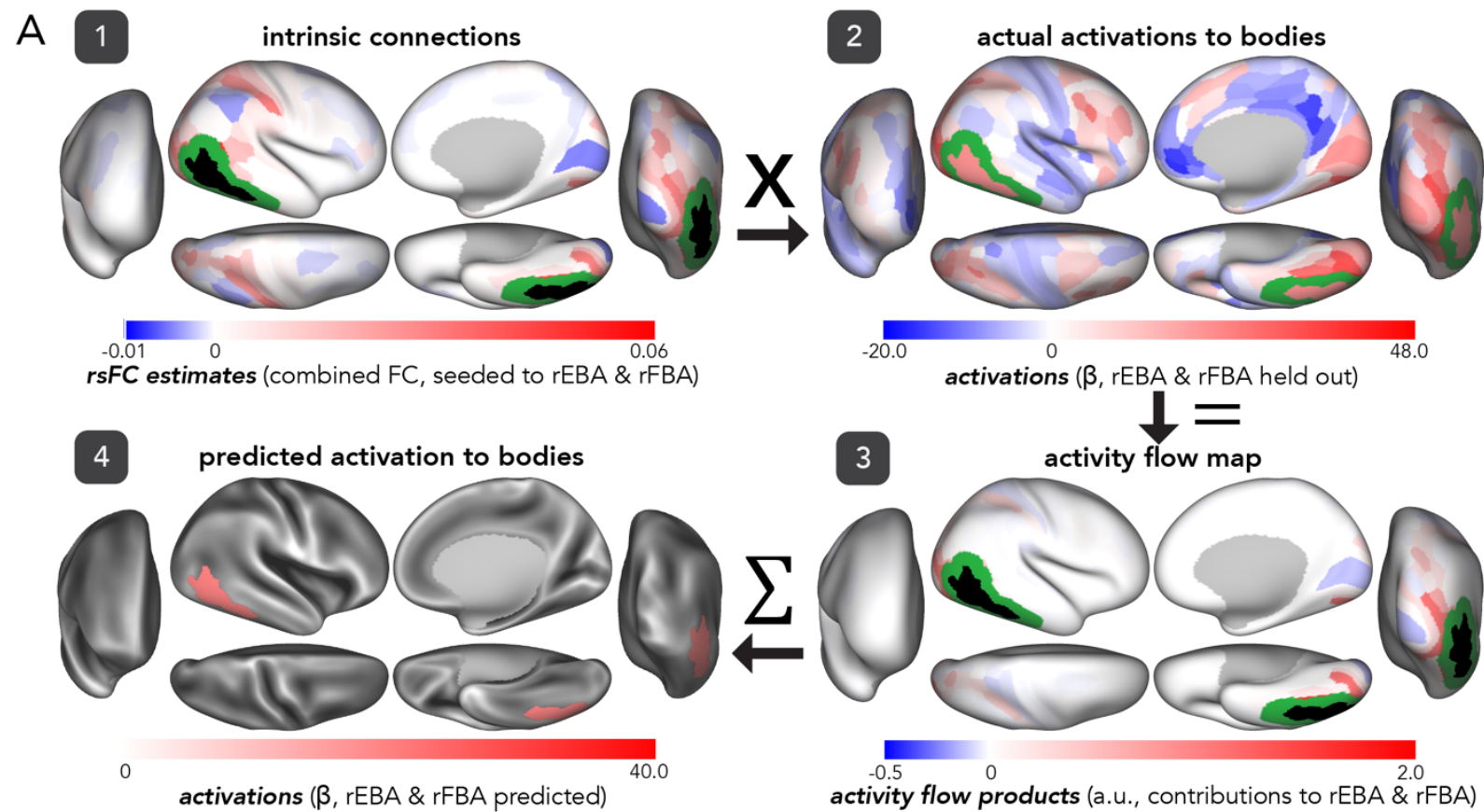

B Connectivity fingerprint (target: rEBA \& rFBA)
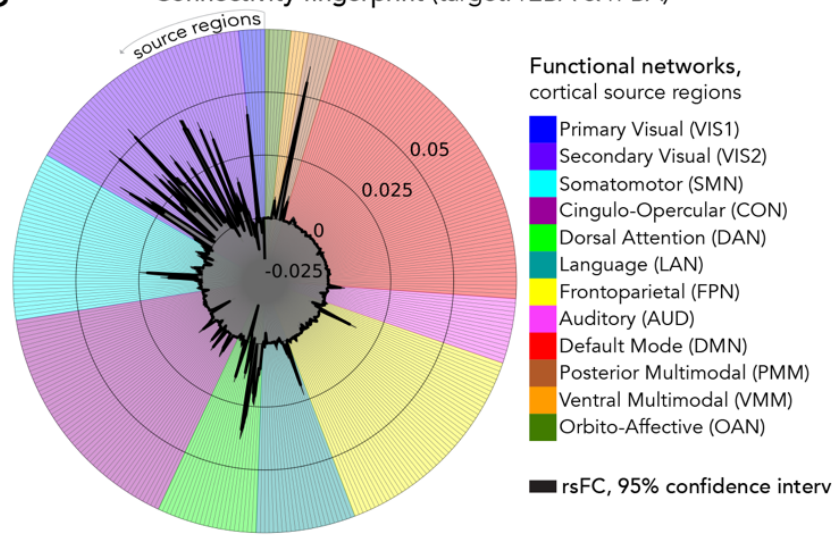

rsFC, $95 \%$ confidence interval

to-be-predicted target region (held out)
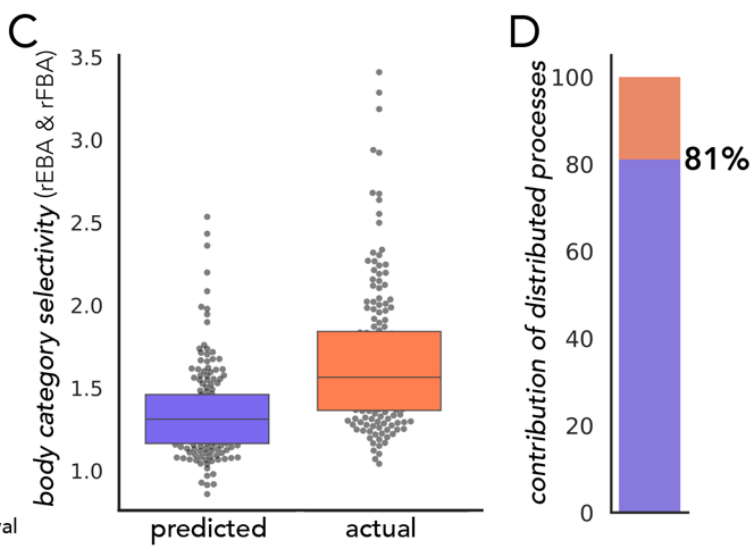

Activity flows for body categories (into: rEBA \& rFBA)

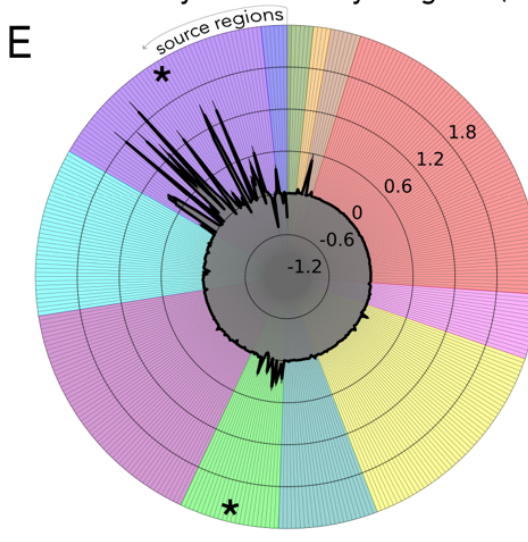

Functional networks, cortical source regions

Primary Visual (VIS1)

Secondary Visual (VIS2) Somatomotor (SMN)

Cingulo-Opercular (CON)

Dorsal Attention (DAN)

Language (LAN)

Frontoparietal (FPN)

Auditory (AUD)

Default Mode (DMN)

Posterior Multimodal (PMM)

Ventral Multimodal (VMM)

Orbito-Affective (OAN)

- flows, $95 \%$ confidence interval

Network contributions to response profile (predicting: rEBA \& rFBA)

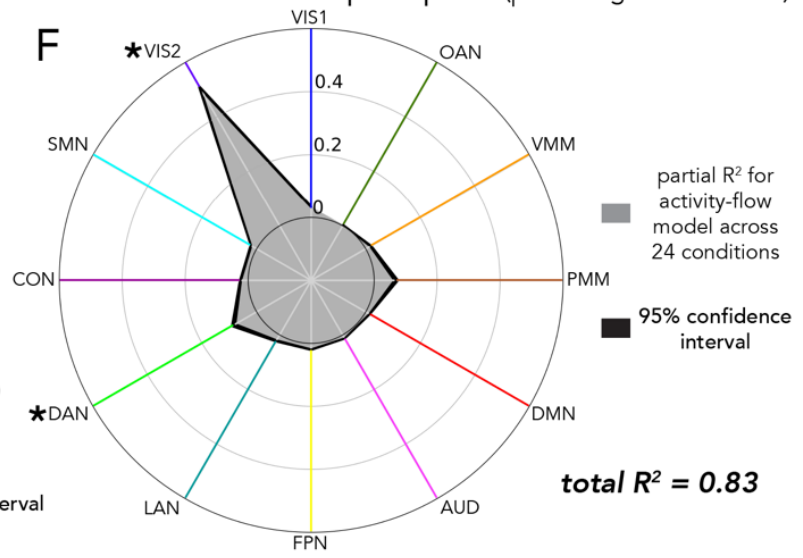


Figure 3. Distributed activity flows account for the majority of localized selectivity to bodies. (A) The activity flow mapping procedure (steps match Methods Fig. 7C) predicting body responses in EBA/FBA (black), projected onto cortical schematics (right hemisphere). Green: source vertices excluded from analyses. Step 2 was not blacked out for visual comparison with step 4, however it was held out in-analysis. Step 4 color scale shows maximum of all regions' predicted activations to body images for visual comparison with step 2. (B) The connectivity fingerprint (Passingham et al., 2002; Mars et al., 2018) of the right EBA/FBA via rsFC (black lines). Radial lines: source regions connected to EBA/FBA, clustered by functional network assignments (Ji et al., 2019) (colored per legend; Fig. 1C). 95\% confidence interval: across participants. (C) Right EBA/FBA body selectivity: activity-flow-predicted (purple) and actual (coral). Grey dots: individual participants' scores. (D) Estimated contribution of distributed processing mechanisms to body selective responses in EBA/FBA. (E) The mapped activity flows (as in A3) of each source region contributing to predicted EBA/FBA responses to body images (statistical significance asterisks at network-mean level). (F) Variance explained by each network-restricted activity flow model (unmixed partial $R^{2}$ in grey) of EBA/FBA's response profile. Black lines: $95 \%$ confidence interval across participants. Asterisks: statistical significance versus each other network. E-F suggests that activity flowing over interactions with VIS2 and DAN represents a general network coding mechanism for EBA/FBA. See Methods for full details of each analysis.

We assessed body selectivity in the EBA/FBA - quantified as the ratio of body to non-body category activations - in both activity-flow-predicted (Fig. 3C, purple) and actual (Fig. 3C, coral) data. As expected, predicted and actual body selectivity were statistically significant relative to a selectivity ratio of 1.0 (i.e., greater than the null hypothesis of equivalent responses to bodies and non-bodies): activity-flow-predicted mean body selectivity $=1.35$, Cohen's $d=1.29$; actual mean body selectivity $=1.67$, Cohen's $d=1.55$; max-T threshold(166) $=5.88, p<0.00001$; Fig. 3C). We next estimated the contribution of distributed processing mechanisms to body selectivity via the percentage of actual body selectivity captured by predicted body selectivity (Fig. 3D). Given that activity flow mapping predicts task-evoked activity based on distributed features (Cole et al., 2016; Fig. 7B), this ratio approximates the extent that distributed processes confer body selectivity in the EBA/FBA (relative to local processes and/or error). As expected, the estimated contribution of distributed processing mechanisms was $81 \%$, which was statistically greater than $50 \%$ (see Methods) (Cohen's $d=2.52$, maxT threshold $(166)=6.09, p<0.00001$; Fig. 3D) (left hemisphere and replication: Table S3).

Next, we examined how each large-scale functional network (Fig. 1C; Ji et al., 2019) contributed to the observation that activity flowing over a distributed network (connectivity fingerprint: Fig. 3B) shaped body selectivity in the EBA/FBA. The activity flows map (Fig. 3A, step 3) — where source activity was weighted by rsFC with the (held out) target region - was an entry point to assess such network contributions. Firstly, we averaged the estimated activity flows (i.e., source region contributions to predicted body activations of the EBA/FBA) based on network assignment (Fig. 3E). Secondly, we used dominance analysis to identify each functional network's unmixed contribution (partial 
$\mathrm{R}^{2}$; Methods) to the activity-flow-predicted response profile (i.e., activations across all 24 conditions; Fig. 1D) of the EBA/FBA (Fig. 3F). The secondary visual network (VIS2) activity flows exhibited the largest contribution (versus each other network) to EBA/FBA's bodyevoked activations (Fig. 3E; max-T threshold (175) = 3.39, $p<0.0001$; left hemisphere and replication: Table S4). Dorsal attention network (DAN) activity flows were also significantly higher (same max-T thresholds; Methods) than most other networks, except VIS2 and posterior multimodal network (PMM). VIS2 also accounted for most of the explained variance in EBA/FBA's response profile (partial $R^{2}=0.51,61 \%$ of the full model $\mathrm{R}^{2}$; Fig. 3F). DAN accounted for the next highest amount of explained variance (partial $\mathrm{R}^{2}=0.09,10 \%$ of the full model $\mathrm{R}^{2}$, Fig. 3F; left hemisphere and replication: Tables S5S7). Thus, for both body-specific conditions and across all conditions (response profile), activity flowing over VIS2 and DAN exhibited substantial contributions to EBA/FBA processing.

As hypothesized, we found evidence that activity flowing over functional connections shaped body selectivity in the EBA/FBA. Results also suggested that distributed processing mechanisms chiefly specified body-selective responses in the EBA/FBA. The total explained variance in the EBA/FBA's 24-condition response profile was significantly greater than $50 \%$ (total $R^{2}=0.83$, versus 0.5 : $t(175)=66.07, p=1.14 x$ 10-125; left hemisphere and replication: Tables S5-S7), suggesting that distributed processes predominantly influence EBA/FBA responses across a variety of cognitive domains.

\section{Distributed processes contribute to face category selectivity}

The fusiform face area (FFA) is a hallmark region exhibiting localized category selectivity given its well-replicated relationship with face processing (Kanwisher et al., 1997; Kanwisher and Yovel, 2006). The posterior superior temporal sulcus (pSTS) is thought to be a multisensory processing region responsive to the changeable aspects of faces (Puce et al., 1998; Osher et al., 2016). The FFA and pSTS are loci of specialization in a two pathway model (Baseler et al., 2014; Li et al., 2019), representing facial identity (relatively invariant) and facial expression (dynamic), respectively (Haxby et al., 2001; Pitcher et al., 2020). While both the FFA and pSTS are part of the VTC, the pSTS is more dorsal and lies adjacent to the temporoparietal junction; the FFA is more posterior and typically identified on the ventral aspect of the cortex (i.e., the fusiform gyrus) (Fig. 2B). Extending our core hypothesis to the processing of face images, we hypothesized that activity flow via the connectivity fingerprint of the FFA and pSTS (Fig. 4A-B) (henceforth: FFA/pSTS) is likely the primary determinant of its face selective responses. 
bioRxiv preprint doi: https://doi.org/10.1101/2022.02.19.481103; this version posted February 22, 2022. The copyright holder for this preprint (which was not certified by peer review) is the author/funder, who has granted bioRxiv a license to display the preprint in perpetuity. It is made available under aCC-BY-NC-ND 4.0 International license.

A
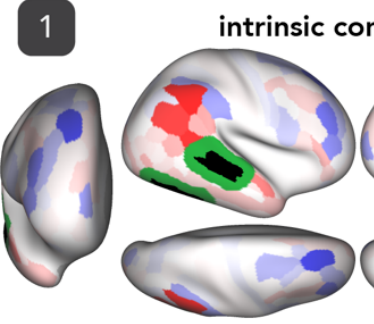

$-0.01 \quad 0$

rsFC estimates (combined FC, seeded to rFFA \& rpSTS)

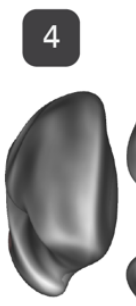

predicted activation to faces
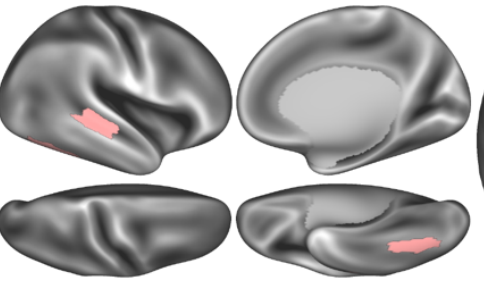

0

activations ( $\beta$, rFFA \& rpSTS predicted)

source vertices excluded from analysis

B
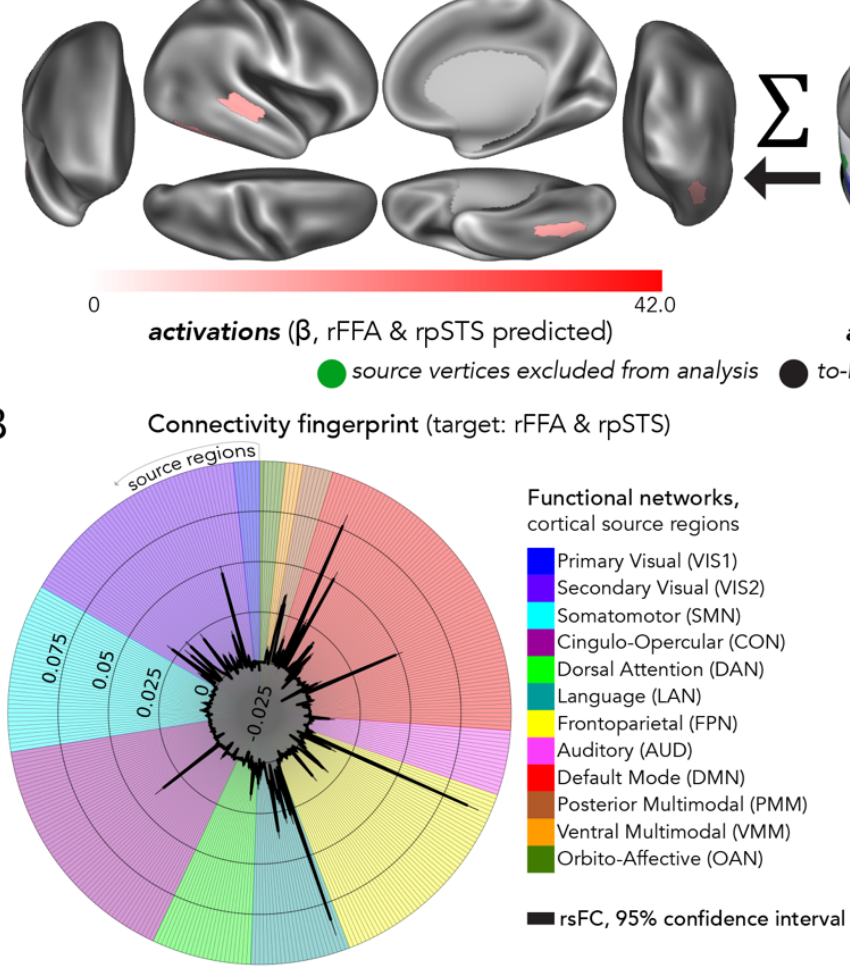

2

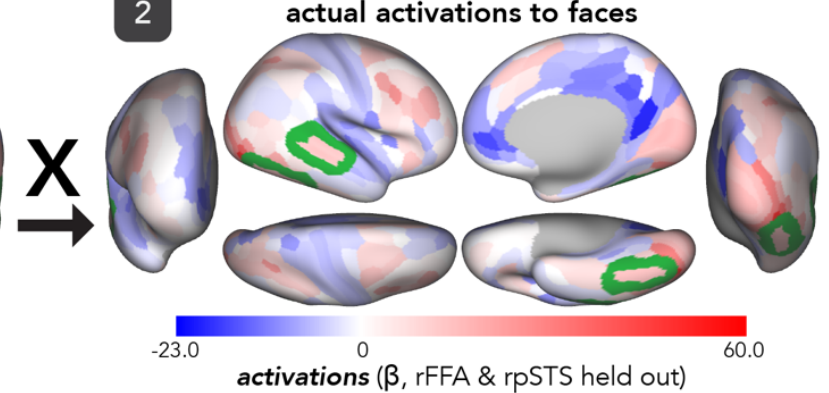

$\downarrow=$

3 activity flow map

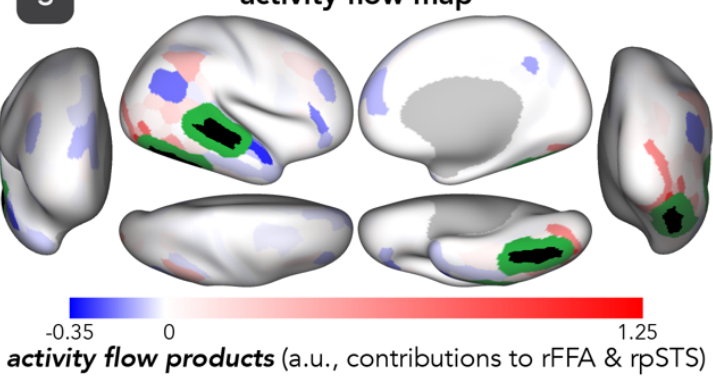

activity flow products (a.u., contributions to rFFA \& rpSTS)

to-be-predicted target region (held out)
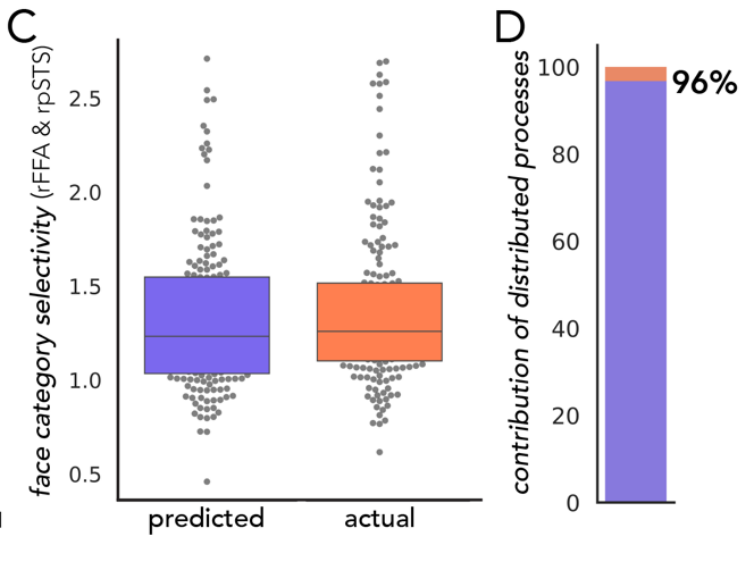

Activity flows for face categories (into: rFFA \& rpSTS)

$E$

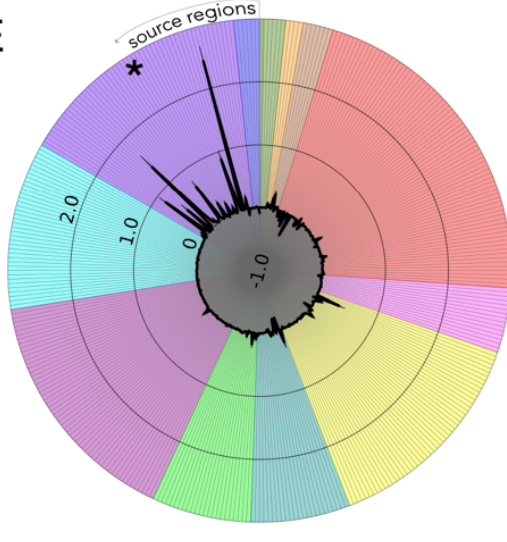

Auditory (AUD)
Functional networks, cortical source regions

Primary Visual (VIS1)

Secondary Visual (VIS2)

Somatomotor (SMN)

Cingulo-Opercular (CON)

Dorsal Attention (DAN)

Language (LAN)

Frontoparietal (FPN)

Default Mode (DMN)

Posterior Multimodal (PMM)

Ventral Multimodal (VMM)

Orbito-Affective (OAN)

flows, $95 \%$ confidence interval
Network contributions to response profile (predicting: rFFA \& rpSTS)

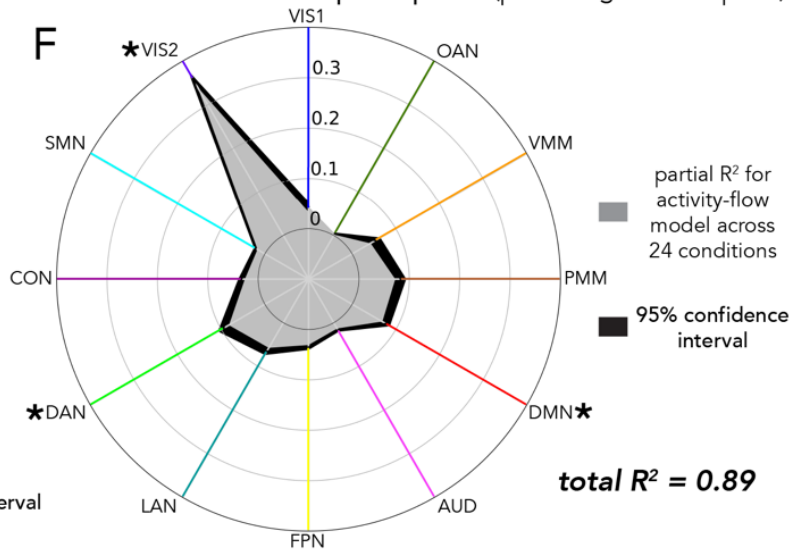


Figure 4. Distributed activity flows account for the majority of localized selectivity to faces. All figure specifications follow Fig. 3. (A) The activity flow mapping procedure predicting activations to face categories, projected onto cortical schematics (right hemisphere). Right FFA/pSTS was the held out target complex. (B) The connectivity fingerprint (Passingham et al., 2002; Mars et al., 2018) of the right FFA/pSTS via whole-cortex rsFC (black lines). Radial lines: source regions connected to the FFA/pSTS, clustered by functional network assignments (Ji et al., 2019) (colored per legend and Fig. 1C). (C) Face category selectivity exhibited by the right FFA/pSTS. (D) Estimated contribution of distributed processing mechanisms to face selectivity exhibited by the right FFA/pSTS. (E) Mapped activity flows (as in A step 3) of each source region contributing to the prediction of FFA/PSTS responses to face images. VIS2 regions contributed most to the FFA/pSTS predicted activation magnitude to faces. (F) Variance explained by each network-restricted activity flow model (unmixed partial $R^{2}$ via dominance analysis; see Methods) of the right FFA/pSTS' response profile. VIS2 accounted for the most variance, altogether suggesting that activity flowing over VIS2 regions represents a general network coding mechanism for FFA/pSTS processing. DAN and DMN regions also accounted for a nontrivial amount of variance at the response-profile level suggesting that, across diverse cognitive domains, FFA/pSTS processing is impacted by activity flowing over DAN and DMN regions, in addition to VIS2 (in the face-specific case).

We followed the aforementioned pipeline for body selectivity exactly, except analyzing face images and the FFA/pSTS (Fig. 4A; black regions). Overall, as expected, the pattern of results observed for body processing (EBA/FBA) extended to face processing (FFA/pSTS). Face selectivity was observed in both the actual and activityflow-predicted data (predicted mean face selectivity $=1.33$, Cohen's $d=0.83$; actual mean face selectivity $=1.37$, Cohen's $d=0.89$; max-T threshold $(170)=7.35, p<$ 0.00001 ; Fig. 4C), and the estimated contribution of distributed processing mechanisms was 96\% (Cohen's $d=2.27$, max-T threshold $(170)=4.83, p<0.00001$; Fig. 4D) (left hemisphere and replication: Table S3). We found that face activations in the FFA/pSTS were most influenced by activity flows over VIS2 connections (max-T(175) $=3.39$, $p<0.0001$; Fig. 4E; left hemisphere and replication: Table S4). VIS2 also accounted for the most variance in FFA/PSTS processing at the response profile level (40\%; left hemisphere and replication: Tables S5-S7).

These results suggest that face selectivity in the FFA/pSTS was significantly shaped by activity flow processes over a distributed network architecture, as hypothesized. These distributed processes were most prominently influenced by activity flowing over VIS2, similar to the EBA/FBA. Across all conditions, activity flowing over dorsal attention (DAN) and default mode (DMN) network connections were predictive as well (DAN: 11\%, DMN: 9\%). The total explained variance in this response profile model (Fig. 4F) was $\mathrm{R}^{2}=0.89$ (versus $0.5: t(175)=92.61, p=1.32 \times 10^{-150}$; left hemisphere and replication: Tables S5-S7), suggesting that distributed processes chiefly influenced FFA/pSTS responses across many cognitive domains.

Distributed processes contribute to place category selectivity 
The next visual category contained place images (Barch et al., 2013), sometimes termed scenes, environment, or topography. Place-specific regions include the parahippocampal place area (PPA) and the retrosplenial cortex (RSC) (Fig. 2C), which are thought to act cooperatively toward a cohesive percept (Epstein and Higgins, 2007; Park and Chun, 2009; Sulpizio et al., 2014). The PPA is thought to compute viewpoint-specific discrimination (Epstein et al., 2003), as well as mediating (or binding) contextual associations pertinent for episodic memory (Aminoff et al., 2013). The RSC is thought to provide the medial temporal lobe with visuospatial information (Kravitz et al., 2011), and to integrate viewpoint-invariant information for navigation and learning (Park and Chun, 2009). The PPA consisted of previously identified parcels (Weiner et al., 2018), and the dorsal RSC corresponded to Brodmann areas 29 and 30 (Vann et al., 2009) (Fig. 2C). Extending our core hypothesis to the processing of place images, we hypothesized that activity flow via the connectivity fingerprint of the PPA and RSC (Fig. 5A-B) (henceforth: PPA/RSC) is likely the primary determinant of its place-selective responses. 
bioRxiv preprint doi: https://doi.org/10.1101/2022.02.19.481103; this version posted February 22, 2022. The copyright holder for this preprint (which was not certified by peer review) is the author/funder, who has granted bioRxiv a license to display the preprint in perpetuity. It is made available under aCC-BY-NC-ND 4.0 International license.

A

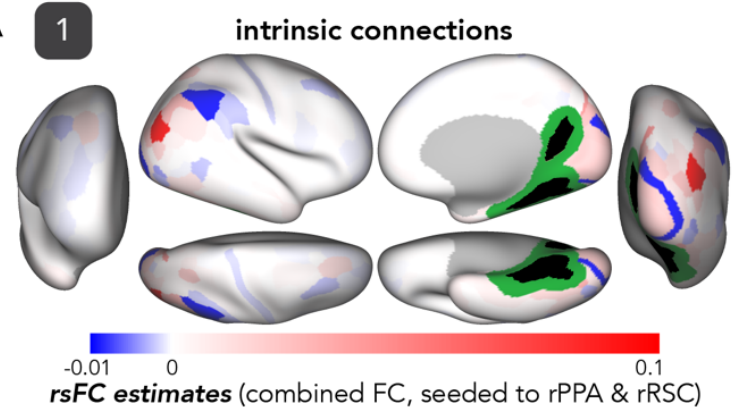

rsFC estimates (combined FC, seeded to rPPA \& rRSC)

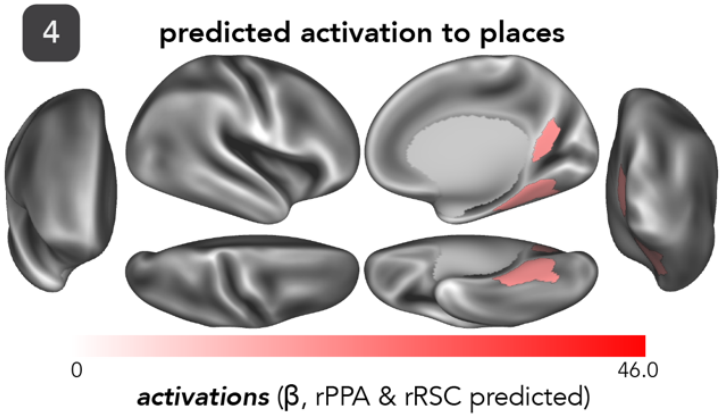

source vertices excluded from analysis

B

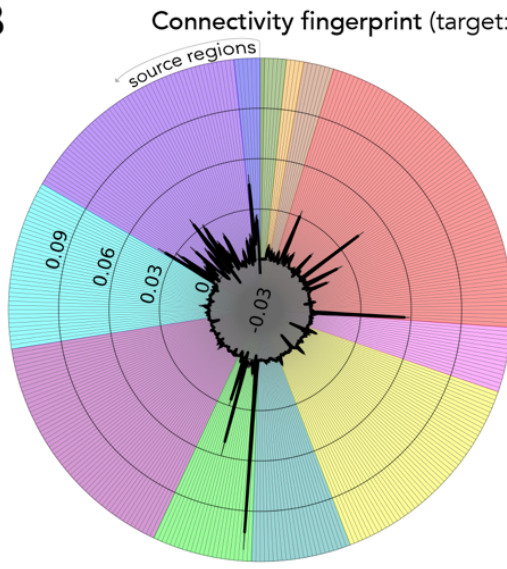

Activity flows for place categories (into: rPPA \& rRSC)
E

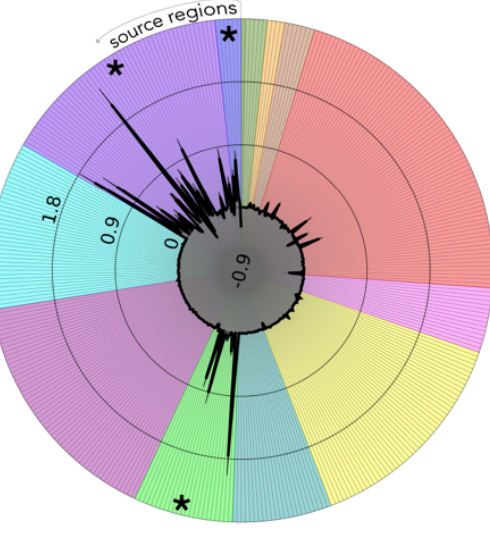

Functional networks, cortical source regions

Primary Visual (VIS1)

Secondary Visual (VIS2)

Somatomotor (SMN)

Cingulo-Opercular (CON)

Dorsal Attention (DAN)

Language (LAN)

Frontoparietal (FPN)

Auditory (AUD)

Default Mode (DMN)

Posterior Multimodal (PMM)

Ventral Multimodal (VMM)

Orbito-Affective (OAN)

- rsFC, $95 \%$ confidence interval
2 actual activations to places

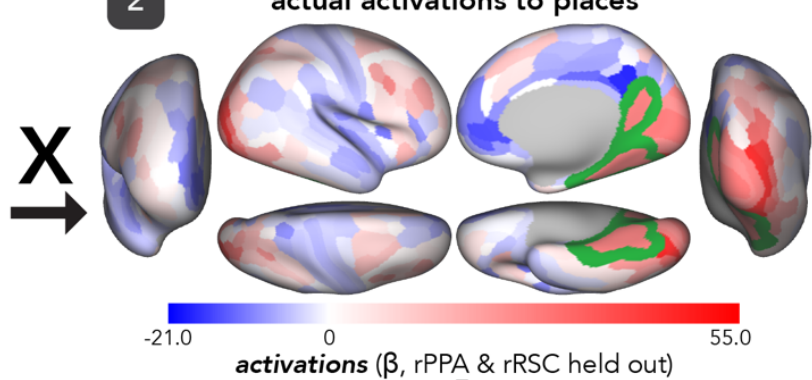

$\downarrow=$

3 activity flow map

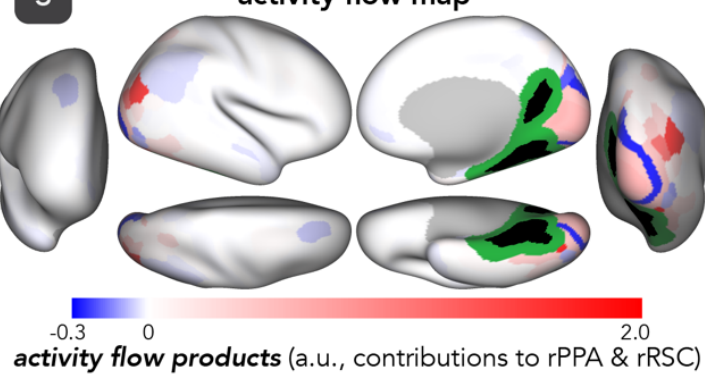

to-be-predicted target region (held out)
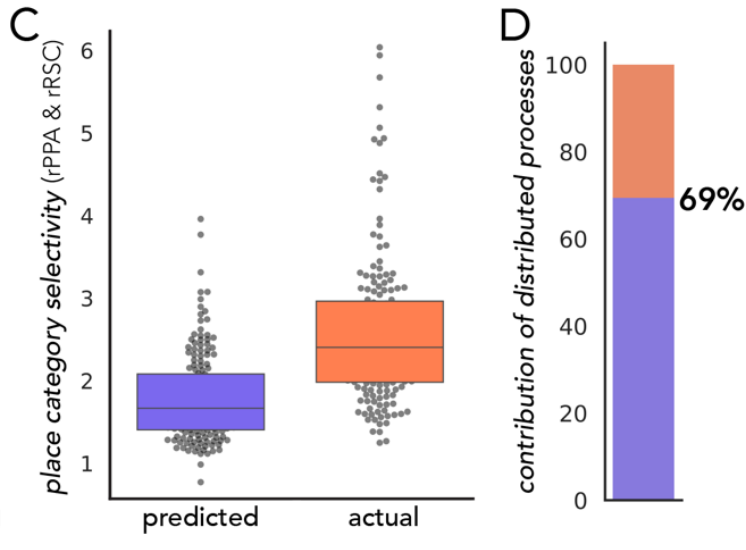

Functional networks, cortical source regions

Primary Visual (VIS1)

Secondary Visual (VIS2) Somatomotor (SMN)

Cingulo-Opercular (CON)

Dorsal Attention (DAN)

Language (LAN)

Frontoparietal (FPN)

Auditory (AUD)

Default Mode (DMN)

Posterior Multimodal (PMM)

Ventral Multimodal (VMM)

Orbito-Affective (OAN)

- flows, $95 \%$ confidence interval

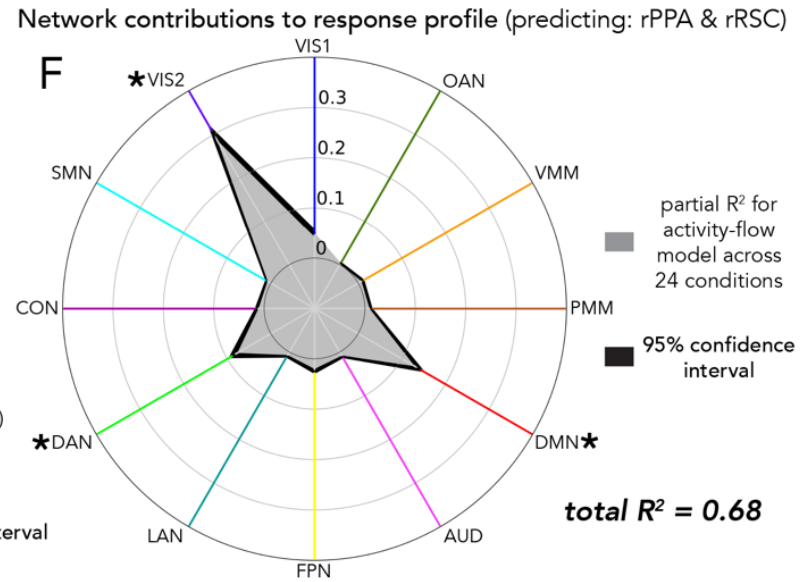


Figure 5. Distributed activity flows account for the majority of localized selectivity to places. All formatting and figure specifications follow Fig. 3. (A) The activity flow mapping procedure to predict activations to place categories, projected onto cortical schematics (right hemisphere only). Right PPA/RSC was the held out target complex. (B) The connectivity fingerprint of the right PPA/RSC, as in Passingham et al. (2002), except with whole-cortex rsFC (black lines). Radial lines: source regions connected to the PPA/RSC, clustered by CAB-NP functional network assignments (Ji et al., 2019) (colored per legend and Fig. 1C). (C) Place category selectivity exhibited by the PPA and RSC in the right hemisphere. (D) Estimated contribution of distributed processing mechanisms to the emergence of place selective responses in the right PPA/RSC. (E) Mapped activity flows (as in A step 3) of each source region contributing to the prediction of PPA/RSC responses to place images. VIS1, VIS2 and DAN contributed most to the right PPA/RSC predicted activation magnitude to place categories. Note that VIS1, VIS2, and DAN were all statistically greater than each other network, except for each other. (F) Variance explained by each network-restricted activity flow model (partial $R^{2}$ via dominance analysis; see Methods) of the right PPA/RSC's response profile. VIS2, DAN, and DMN accounted for the most variance, suggesting that activity flowing over regions in these networks represents a general network coding mechanism for PPA and RSC processing, while VIS1 contributes to place-specific responses.

Significant place selectivity was observed in the PPA/RSC (predicted mean place selectivity $=1.8$, Cohen's $d=1.49$; actual mean place selectivity $=2.59$, Cohen's $d=$ 1.74; max-T threshold (166) $=3.08, p<0.00001 ;$ Fig. 5C), and the estimated contribution of distributed processes was 69\% (versus 50\%: Cohen's $d=1.15$, max-T threshold (166) $=6.88, p<0.00001 ;$ Fig. 5D) (left hemisphere and replication: Table S3). Activity flowing over VIS1, VIS2, and DAN provided the largest contributions to PPA/RSC's place activations (all significant except when compared to each other: max- $T$ threshold (175) $=$ 3.4, $p<0.0001$; Fig. 5E) (left hemisphere and replication: Table S4). A similar set of networks accounted for the most variance at the response profile level (Fig. 5F), including VIS2 (44\%), DAN (13\%) and DMN contributions (21\%) (left hemisphere and replication: Tables S5-S7).

As hypothesized, the connectivity fingerprint of the PPA/RSC significantly shaped its place-selectivity. PPA/RSC responses were influenced by activity flowing over VIS2 and DAN. Additionally, VIS1 was particularly important for place-specific activity; and DMN for cross-domain activity (Fig. 5E-F), suggesting that PPA/RSC's activity flow processes were more heterogeneous than prior models. The total variance in the PPA/RSC response profile explained by activity flow processes (Fig. 5F) was greater than $50 \%$ (total $R^{2}=0.68 ; t(175)=23.93, p=4.51 \times 10^{-57}$; left hemisphere and replication: Tables S5-S7), which provides evidence that distributed processes predominantly influenced PPA/RSC activations to a variety of cognitive domains.

Distributed processes contribute to tool category selectivity 
The final visual category included tool images (Barch et al., 2013), sometimes termed inanimate objects. Following an extensive literature on object recognition (Grill-Spector et al., 2001; Bar, 2004), we hypothesized that tool selectivity is exhibited by the lateral occipital complex (LOC) (Beauchamp et al., 2002; Osher et al., 2016), which is posteriorly located and wraps around the cortex in the ventromedial direction (Fig. 2D). The LOC is thought to represent higher-level information of objects, as opposed to low level visual features (Kourtzi and Kanwisher, 2001), suggesting a role at the category level of visual processing. However, reports vary on the degree of semantic content processed by the LOC (Victoria et al., 2019). Thus, the link between the LOC and tool selectivity was the least clear (of all four models) a priori. Extending our core hypothesis to the processing of tool images, we hypothesized that activity flow via the connectivity fingerprint of the LOC (Fig. 6A-B) is likely the primary determinant of its tool selective responses. 
bioRxiv preprint doi: https://doi.org/10.1101/2022.02.19.481103; this version posted February 22, 2022. The copyright holder for this preprint (which was not certified by peer review) is the author/funder, who has granted bioRxiv a license to display the preprint in perpetuity. It is made available under aCC-BY-NC-ND 4.0 International license.

A

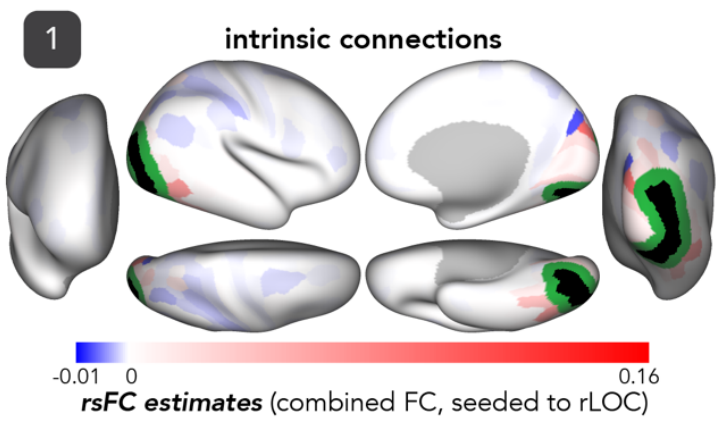

4

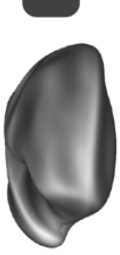

predicted activation to tools

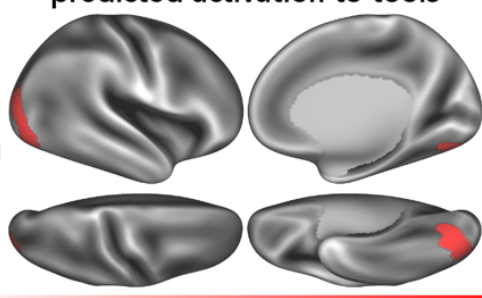

0

activations ( $\beta$, rLOC predicted)

source vertices excluded from analysis

B

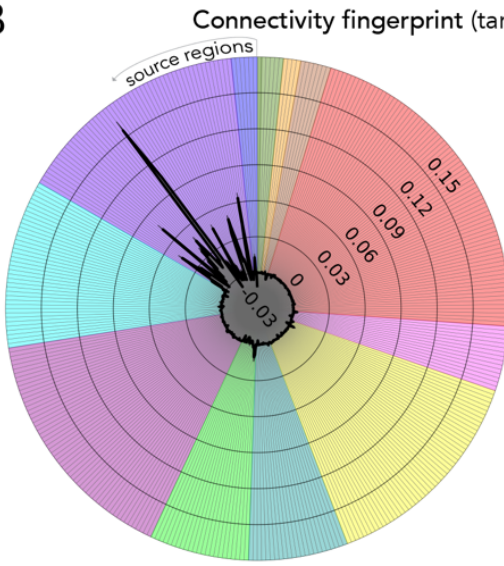

Functional networks, cortical source regions

Primary Visual (VIS1)

Secondary Visual (VIS2)

Somatomotor (SMN)

Cingulo-Opercular (CON)

Dorsal Attention (DAN)

Language (LAN)

Frontoparietal (FPN)

Auditory (AUD)

Default Mode (DMN)

Posterior Multimodal (PMM)

Ventral Multimodal (VMM)

Orbito-Affective (OAN)

- rsFC, $95 \%$ confidence interval

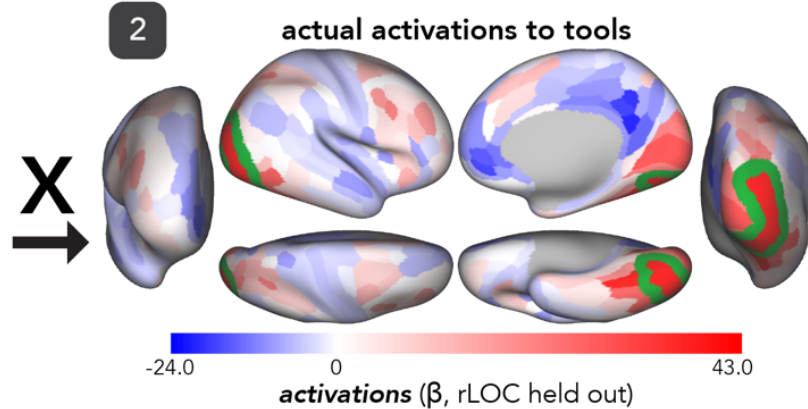

$\downarrow=$

$3 \quad$ activity flow map

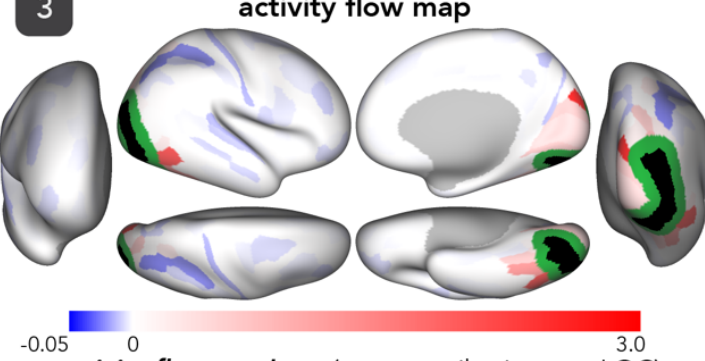

activity flow products (a.u., contributions to rLOC)
C

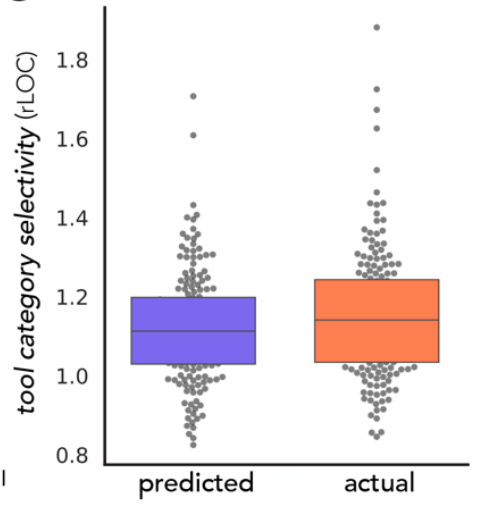

D

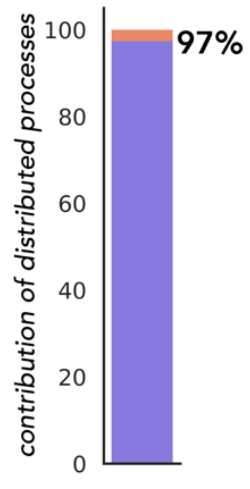

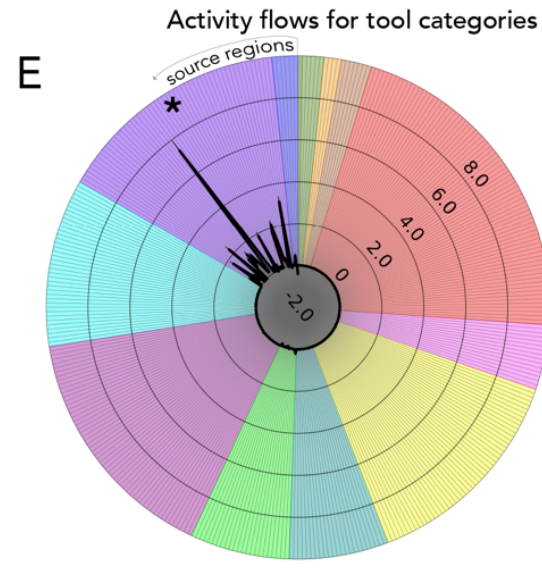

Functional networks, cortical source regions

Primary Visual (VIS1)

Secondary Visual (VIS2)

Somatomotor (SMN)

Cingulo-Opercular (CON)

Dorsal Attention (DAN)

Language (LAN)

Frontoparietal (FPN)

Auditory (AUD)

Default Mode (DMN)

Posterior Multimodal (PMM)

Ventral Multimodal (VMM)

Orbito-Affective (OAN)

- flows, $95 \%$ confidence interval

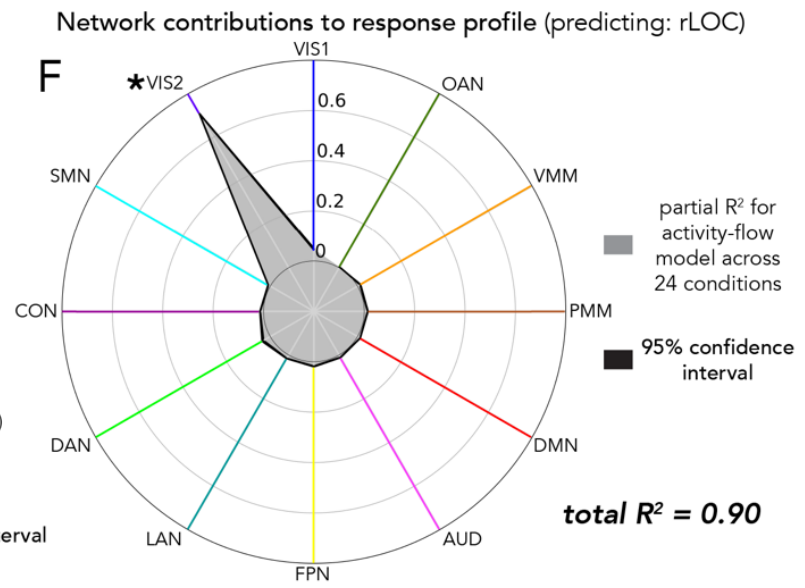


Figure 6. Distributed activity flows account for the majority of localized selectivity to tools. All formatting and figure specifications as in Fig. 3. (A) The activity flow mapping procedure to predict activations to tool categories in the held out target - the right LOC - projected onto cortical schematics (right hemisphere). (B) The connectivity fingerprint of the right LOC, as in Passingham et al. (2002), except with whole-cortex rsFC (black lines). Radial lines: source regions connected to the LOC, clustered by CAB-NP functional network assignments (Ji et al., 2019) (colored per legend and Fig. 1C). (C) Tool category selectivity exhibited by the LOC in the right hemisphere. (D) Estimated contribution of distributed processing mechanisms to the emergence of tool selective responses in the right LOC. (E) Mapped activity flows (as in A step 3) of each source region contributing to the prediction of LOC responses to tool images. VIS2 contributed most to the right LOC predicted activation magnitude to tool categories (F) Variance explained by each network-restricted activity flow model (partial $\mathrm{R}^{2}$ via dominance analysis; see Methods) of the right LOC's response profile. VIS2 accounted for the most variance, suggesting that activity flowing over regions in these networks represents a network coding mechanism for LOC processing.

Tool selectivity in the LOC was statistically significant (predicted tool selectivity $=$ 1.12, Cohen's $d=0.88$; actual tool selectivity $=1.15$, Cohen's $d=0.95$; max- $T$ threshold $(174)=9.61, p<0.00001$; Fig. 6C), and the estimated contribution of distributed processes to tool selectivity in the LOC was particularly high at $98 \%$ (Cohen's $d=8.45$, max-T threshold (174) $=5.15$; Fig. 6D) (left hemisphere and replication: Table S3). VIS2 activity flows demonstrated a strikingly high contribution to LOC's tool-specific responses $(\max -\mathrm{T}(175)=3.4, p<0.0001 ;$ Fig. 6E) (left hemisphere and replication: Table S4), and accounted for the majority of the variance in LOC's response profile (79\%; Fig. 6F) (left hemisphere and replication: Tables S5-S7).

As hypothesized, tool selectivity was strongly influenced by distributed activity flow processes (97-98\%). As in all other models (bodies, faces, and places) the total explained variance that activity flow mapping captured for the LOC response profile was greater than $50 \%$ (total $R^{2}=0.90$ vs $0.5: t(175)=115.55, p=3.71 \times 10^{-167}$ ). Given that activity flow mapping is a distributed processing model (Methods Fig. 7B), this suggests that distributed processes represent a general mechanism in LOC activations.

\section{Control analyses: null connectivity fingerprints reduce visual category selectivity}

To corroborate that each functional complex's unique connectivity fingerprint (Figs. 3B6B) - its placement in the brain's network architecture - determined its visual category selectivity (Figs. 3C-6C), we built null models based on FC substitution. We hypothesized that a null connectivity fingerprint (i.e., a fingerprint based on the wrong functional complex) would confer significantly lower activity-flow-predicted category selectivity. 
Table 1. Null model connectivity fingerprints do not generate visual category selective responses in functional complexes.

\begin{tabular}{|c|c|c|c|}
\hline Comparison & $t(175)$ & $p$-value & Cohen's d \\
\hline \multicolumn{4}{|l|}{$\begin{array}{l}\text { True model body selectivity > null model body selectivity when } \\
\text { EBA/FBA rsFC was substituted with: }\end{array}$} \\
\hline face complex connectivity fingerprint: FFA/pSTS rsFC & 6.36 & $1.7 \times 10^{-9}$ & 0.61 \\
\hline place complex connectivity fingerprint: PPA/RSC rsFC & 3.53 & 0.0005 & 0.37 \\
\hline tool complex connectivity fingerprint: LOC rsFC & 11.34 & $1.1 \times 10^{-22}$ & 1.16 \\
\hline \multicolumn{4}{|l|}{$\begin{array}{l}\text { True model face selectivity > null model face selectivity when } \\
\text { FFA/pSTS rsFC was substituted with: }\end{array}$} \\
\hline body complex connectivity fingerprint: EBA/FBA rsFC & 8.21 & $4.7 \times 10^{-14}$ & 0.81 \\
\hline place complex connectivity fingerprint: PPA/RSC rsFC & 21.45 & $7.1 \times 10^{-51}$ & 2.11 \\
\hline tool complex connectivity fingerprint: LOC rsFC & 14.95 & $4.4 \times 10^{-33}$ & 1.48 \\
\hline \multicolumn{4}{|l|}{$\begin{array}{l}\text { True model place selectivity > null model place selectivity when } \\
\text { PPA/RSC rsFC was substituted with: }\end{array}$} \\
\hline body complex connectivity fingerprint: EBA/FBA rsFC & 14.02 & $2.1 \times 10^{-30}$ & 1.50 \\
\hline face complex connectivity fingerprint: FFA/pSTS rsFC & 14.46 & $1.1 \times 10^{-31}$ & 1.44 \\
\hline tool complex connectivity fingerprint: LOC rsFC & 11.87 & $2.3 \times 10^{-24}$ & 1.20 \\
\hline \multicolumn{4}{|l|}{$\begin{array}{l}\text { True model tool selectivity > null model tool selectivity when LOC } \\
\text { rsFC was substituted with: }\end{array}$} \\
\hline body complex connectivity fingerprint: EBA/FBA rsFC & 2.74 & 0.007 & 0.22 \\
\hline face complex connectivity fingerprint: FFA/pSTS rsFC & 9.97 & $7.8 \times 10^{-19}$ & 0.91 \\
\hline place complex connectivity fingerprint: PPA/RSC rsFC & 2.06 & 0.04 & 0.22 \\
\hline
\end{tabular}

As hypothesized, visual category selectivities were significantly greater when based upon the true connectivity fingerprints (Table 1). This assessment is more stringent than a null model that randomly scrambles FC because there is likely some similarity in visual processing (and therefore activity flow patterns) across the four functional complex's network interactions. However, no substituted connectivity fingerprint was sufficiently similar to the true fingerprint to generate the original visual category selectivity. Each of these results were corroborated in the left hemisphere and in the replication dataset (Tables S8-S9). These results corroborate that the connectivity fingerprint unique to each functional complex significantly shaped its visual category selective response. 


\section{Discussion}

The foregoing results suggest that local category selectivity in classically studied regions of visual cortex is generated primarily by distributed activity flowing over functional connections (Figs. 3A-6A), supporting a central tenet of the proposed distributed convergence theory. We observed significant visual category selectivity in four functional complexes for both actual and activity-flow-predicted responses (Figs. 3C-6C). Further, modeled distributed processing mechanisms accounted for the majority of variance in visual category selectivity in all four complexes (Figs. 3D-6D). Across all assessments, VIS2 interactions had the most prominent contribution to both category-specific and response profile processes. Three categories exhibited additional contributions by other networks: body-EBA/FBA activity from DAN; face-FFA/pSTS from DAN and DMN; and place-PPA/RSC from DAN, VIS1, and DMN (Figs. 3-6E-F). Lastly, activity flow mapping based on swapping connectivity fingerprints between category selective visual regions yielded significantly lower visual category selectivity, demonstrating the importance of each region's unique connectivity fingerprint for generating category selectivity. Altogether, these findings support the hypothesis that connectivity fingerprints of visual cortex regions (Figs. 3B-6B) chiefly shape their category selective responses.

These findings build upon the notion of distributed and overlapping representations in the visual cortex (Haxby et al., 2001), as well as the general hypothesis that connectivity fingerprints determine a region's functional repertoire (Passingham et al., 2002; Mars et al., 2018). Results here also extend observations that structural connectivity fingerprints (of the same regions studied here) can be used to predict visual category responses (Osher et al., 2016) by modeling activity flow processes (Cole et al., 2016) over connectivity fingerprints. This goes beyond purely predictive models by adding generative activity flow processes to estimate the allocation of distributed processes contributing to category selectivity. Activity flow mapping (Cole et al., 2016) treats cognitive computations as the local convergence of information propagating over a distributed, functionally-organized system (Ito et al., 2020a). In the present study, we leveraged the substantial evidence for localized processing in the case of category selectivity in visual cortex regions to test the distributed convergence hypothesis, revealing how localized activations are likely generated (in large part) by activity flow processes determined by intrinsic functional connectivity fingerprints.

Importantly, activity flow predictions did not account for all task-evoked activity variance, leaving room for local mechanisms to carry out critical computations. Here we adopted a definition of local mechanism consistent in fMRI and electroencephalography research: local activity reflects local field potentials, which are thought to reflect inputs and local/within-region processing (Logothetis, 2003). Therefore it was not a guarantee that distributed mechanisms would exhibit the predominant influence in this fMRI dataset, but two major lines of evidence support this claim. Firstly, high prediction 
accuracy of activity-flow-mapped visual category responses was observed in all functional complexes. If local mechanisms strictly accounted for these responses we would expect lower prediction accuracy, given prior simulation-based findings demonstrating that successful activity flow mapping requires high distributed processing (global coupling) and low local processing (self-coupling/recurrence) (Methods Fig. 7B; Cole et al., 2016). Predicted category selectivity within this framework is thus a proxy for the degree of distributed mechanisms underlying category selectivity. Secondly, the estimated contribution of distributed processes to category selectivity was significantly greater than $50 \%$ for all functional complexes assessed. We additionally observed that activity flow processes explained the majority of variance in response profiles (across 24 diverse task conditions) exhibited by each complex (Figs. 3F-6F), suggesting that activity flowing over global connectivity patterns is a general mechanism in the emergence of localized fMRI responses. However, large-scale functional networks had differential influence depending on the cognitive domain and target complex. For example, LOC responses exhibited a general, strong influence from VIS2 interactions (Fig. 6E-F), however, FFA/pSTS exhibited strong VIS2 influence in the face-specific model (Fig. 4E) with additional influence from DAN and DMN in the response profile model (Fig. 4F).

The present results shift the plausibility of neural processing hypotheses away from those proposing primarily localized (within-region) processing and toward those proposing primarily distributed (between-region) processing. This shift toward distributed processing follows from our demonstrations that category selectivity in localized brain regions can be generated using only distributed processes: task-evoked activity flowing over connectivity fingerprints. However, while our connectivity estimates address causal confounds better than standard FC measures (Fig. 1A), they lack directional information, limiting our causal inferences. For instance, if ongoing withinregion process variance strongly influenced downstream regions, then this distributed output activity would contribute (in the incorrect causal direction) to the model's prediction of the local processes. This possibility is unlikely, however, since such strongly category selective output would likely drive downstream regions to also exhibit robust category selectivity. However, all (or most) of the strongly category selective regions were included in each functional complex in our analyses, removing them from prediction sources. Regardless, our results specifically rule out hypotheses that predict a substantial role for within-region (e.g., recurrent) processes in driving localized category selectivity in fMRI data, especially when most of that processing remains local (rather than being sent to other regions). For instance, our results rule out the hypothesis that elevated task-evoked face activity in FFA primarily reflects within-region computations (e.g., within-region interactions between neural populations) to detect or identify faces, with the output to other regions indicating whether a face is present. Instead, the present results suggest elevated task-evoked face activity in FFA primarily reflects connectivity- 
selected activity flows summing together weakly category-selective distributed responses to generate highly category-selective localized responses. The same logic applies to the other visual categories and their respective category selective regions. It will be important for future research to disentangle input and output processes to and from visual category selective brain regions in order to further adjudicate between alternate hypotheses regarding the dominance of localized versus distributed processes.

In summary, we observed that distributed processing mechanisms, specified by connectivity fingerprints, are likely the primary contributor to the emergence of category selectivity in visual cortex regions. This built upon a history of theories regarding distributed and overlapping activation patterns (Haxby et al., 2001), connectivity fingerprints specifying regional functioning (Passingham et al., 2002; Mars et al., 2018), and a wealth of literature on locally specialized functioning, together supporting our proposed theory of distributed convergence. We leveraged activity flow mapping's inherent sensitivity to global processing features (Cole et al., 2016) to estimate the contribution of distributed processes upon visual category selectivity. Looking forward, the present findings can facilitate examinations on the generative capacity of local and distributed processing mechanisms by constraining and contextualizing hypotheses.

\section{References (Main Text)}

1. Kanwisher, N. (2010). Functional specificity in the human brain: a window into the functional architecture of the mind. Proceedings of the National Academy of Sciences of the United States of America, 107(25), 11163-11170. https://doi.org/10.1073/pnas.1005062107

2. Schalk, G., Kapeller, C., Guger, C., Ogawa, H., Hiroshima, S., Lafer-Sousa, R., Saygin, Z. M., Kamada, K., \& Kanwisher, N. (2017). Facephenes and rainbows: Causal evidence for functional and anatomical specificity of face and color processing in the human brain. Proceedings of the National Academy of Sciences of the United States of America, 114(46), 12285-12290. https://doi.org/10.1073/pnas.1713447114

3. Op de Beeck, H. P., Haushofer, J., \& Kanwisher, N. G. (2008). Interpreting fMRI data: maps, modules and dimensions. Nature Reviews. Neuroscience, 9(2), 123-135. https://doi.org/10.1038/nrn2314

4. Mur, M., Bandettini, P. A., \& Kriegeskorte, N. (2009). Revealing representational content with pattern-information fMRI--an introductory guide. Social Cognitive and Affective Neuroscience, 4(1), 101-109. https://doi.org/10.1093/scan/nsn044

5. Borra, E., Ichinohe, N., Sato, T., Tanifuji, M., \& Rockland, K. S. (2010). Cortical connections to area TE in monkey: hybrid modular and distributed organization. Cerebral Cortex , 20(2), 257-270. https://doi.org/10.1093/cercor/bhp096

6. Downing, P. E., Jiang, Y., Shuman, M., \& Kanwisher, N. (2001). A cortical area selective for visual processing of the human body. Science, 293(5539), 2470-2473. https://doi.org/10.1126/science.1063414

7. Taylor, J. C., Wiggett, A. J., \& Downing, P. E. (2007). Functional MRI analysis of body and body part representations in the extrastriate and fusiform body areas. Journal of Neurophysiology, 98(3), 16261633. https://doi.org/10.1152/jn.00012.2007 
8. Kanwisher, N., \& Yovel, G. (2006). The fusiform face area: a cortical region specialized for the perception of faces. Philosophical Transactions of the Royal Society of London. Series B, Biological Sciences, 361(1476), 2109-2128. https://doi.org/10.1098/rstb.2006.1934

9. Park, S., \& Chun, M. M. (2009). Different roles of the parahippocampal place area (PPA) and retrosplenial cortex (RSC) in panoramic scene perception. Neurolmage, 47(4), 1747-1756. https://doi.org/10.1016/j.neuroimage.2009.04.058

10. Peelen, M. V., \& Downing, P. E. (2005). Within-subject reproducibility of category-specific visual activation with functional MRI. Human Brain Mapping, 25(4), 402-408.

https://doi.org/10.1002/hbm.20116

11. Tsao, D. Y., Moeller, S., \& Freiwald, W. A. (2008). Comparing face patch systems in macaques and humans. Proceedings of the National Academy of Sciences of the United States of America, 105(49), 19514-19519. https://doi.org/10.1073/pnas.0809662105

12. Corrow, S. L., Dalrymple, K. A., \& Barton, J. J. (2016). Prosopagnosia: current perspectives. Eye and Brain, 8, 165-175. https://doi.org/10.2147/EB.S92838

13. Haxby, J. V., Gobbini, M. I., Furey, M. L., Ishai, A., Schouten, J. L., \& Pietrini, P. (2001). Distributed and overlapping representations of faces and objects in ventral temporal cortex. Science, 293(5539), 2425-2430. https://doi.org/10.1126/science.1063736

14. Finn, E. S., Shen, X., Scheinost, D., Rosenberg, M. D., Huang, J., Chun, M. M., Papademetris, X., \& Constable, R. T. (2015). Functional connectome fingerprinting: identifying individuals using patterns of brain connectivity. Nature Neuroscience, 18(11), 1664-1671. https://doi.org/10.1038/nn.4135

15. Passingham, R. E., Stephan, K. E., \& Kötter, R. (2002). The anatomical basis of functional localization in the cortex. Nature Reviews. Neuroscience, 3(8), 606-616. https://doi.org/10.1038/nrn893

16. Mars, R. B., Passingham, R. E., \& Jbabdi, S. (2018). Connectivity Fingerprints: From Areal Descriptions to Abstract Spaces. Trends in Cognitive Sciences, 22(11), 1026-1037. https://doi.org/10.1016/j.tics.2018.08.009

17. Sporns, O. (2014). Contributions and challenges for network models in cognitive neuroscience. Nature Neuroscience, 17(5), 652-660. https://doi.org/10.1038/nn.3690

18. Friston, K. J. (2011). Functional and effective connectivity: a review. Brain Connectivity, 1(1), 13-36. https://doi.org/10.1089/brain.2011.0008

19. Reid, A. T., Headley, D. B., Mill, R. D., Sanchez-Romero, R., Uddin, L. Q., Marinazzo, D., Lurie, D. J., Valdés-Sosa, P. A., Hanson, S. J., Biswal, B. B., Calhoun, V., Poldrack, R. A., \& Cole, M. W. (2019). Advancing functional connectivity research from association to causation. Nature Neuroscience, 22(11), 1751-1760. https://doi.org/10.1038/s41593-019-0510-4

20. Cole, M. W., Ito, T., Cocuzza, C., \& Sanchez-Romero, R. (2021). The Functional Relevance of TaskState Functional Connectivity. The Journal of Neuroscience: The Official Journal of the Society for Neuroscience, 41(12), 2684-2702. https://doi.org/10.1523/JNEUROSCI.1713-20.2021

21. Osher, D. E., Saxe, R. R., Koldewyn, K., Gabrieli, J. D. E., Kanwisher, N., \& Saygin, Z. M. (2016). Structural Connectivity Fingerprints Predict Cortical Selectivity for Multiple Visual Categories across Cortex. Cerebral Cortex , 26(4), 1668-1683. https://doi.org/10.1093/cercor/bhu303

22. Cole, M. W., Ito, T., Bassett, D. S., \& Schultz, D. H. (2016). Activity flow over resting-state networks shapes cognitive task activations. Nature Neuroscience, 19(12), 1718-1726. https://doi.org/10.1038/nn.4406

23. Cocuzza, C. V., Sanchez-Romero, R., \& Cole, M. W. (2022). Protocol for activity flow mapping of neurocognitive computations using the Brain Activity Flow Toolbox. STAR Protocols, 3(1), 101094. https://doi.org/10.1016/j.xpro.2021.101094 
24. Pajani, A., Kouider, S., Roux, P., \& de Gardelle, V. (2017). Unsuppressible Repetition Suppression and exemplar-specific Expectation Suppression in the Fusiform Face Area. Scientific Reports, 7(1), 160. https://doi.org/10.1038/s41598-017-00243-3

25. O'Reilly, R. C. (2001). Generalization in interactive networks: the benefits of inhibitory competition and Hebbian learning. Neural Computation, 13(6), 1199-1241. https://doi.org/10.1162/08997660152002834

26. Kriegeskorte, N., \& Douglas, P. K. (2019). Interpreting encoding and decoding models. Current Opinion in Neurobiology, 55, 167-179. https://doi.org/10.1016/j.conb.2019.04.002

27. Ito, T., Hearne, L., Mill, R., Cocuzza, C., \& Cole, M. W. (2020a). Discovering the Computational Relevance of Brain Network Organization. Trends in Cognitive Sciences, 24(1), 25-38. https://doi.org/10.1016/j.tics.2019.10.005

28. Van Essen, D. C., Smith, S. M., Barch, D. M., Behrens, T. E. J., Yacoub, E., Ugurbil, K., \& WU-Minn HCP Consortium. (2013). The WU-Minn Human Connectome Project: an overview. Neurolmage, 80, 62-79. https://doi.org/10.1016/j.neuroimage.2013.05.041

29. Sanchez-Romero, R., \& Cole, M. W. (2021). Combining Multiple Functional Connectivity Methods to Improve Causal Inferences. Journal of Cognitive Neuroscience, 33(2), 180-194. https://doi.org/10.1162/jocn a 01580

30. Norman-Haignere, S. V., McCarthy, G., Chun, M. M., \& Turk-Browne, N. B. (2012). Categoryselective background connectivity in ventral visual cortex. Cerebral Cortex , 22(2), 391-402. https://doi.org/10.1093/cercor/bhr118

31. Li, J., Song, Y., \& Liu, J. (2019). Functional connectivity pattern in the core face network reflects different mechanisms of holistic face processing measured by the whole-part effect and compositeface effect. Neuroscience, 408, 248-258. https://doi.org/10.1016/j.neuroscience.2019.04.017

32. Wierenga, C. E., Perlstein, W. M., Benjamin, M., Leonard, C. M., Rothi, L. G., Conway, T., Cato, M. A., Gopinath, K., Briggs, R., \& Crosson, B. (2009). Neural substrates of object identification: Functional magnetic resonance imaging evidence that category and visual attribute contribute to semantic knowledge. Journal of the International Neuropsychological Society: JINS, 15(2), 169-181. https://doi.org/10.1017/S1355617709090468

33. Op de Beeck, H. P., Pillet, I., \& Ritchie, J. B. (2019). Factors Determining Where Category-Selective Areas Emerge in Visual Cortex. Trends in Cognitive Sciences, 23(9), 784-797. https://doi.org/10.1016/j.tics.2019.06.006

34. Ji, J. L., Spronk, M., Kulkarni, K., Repovš, G., Anticevic, A., \& Cole, M. W. (2019). Mapping the human brain's cortical-subcortical functional network organization. Neurolmage, 185, 35-57. https://doi.org/10.1016/j.neuroimage.2018.10.006

35. Barch, D. M., Burgess, G. C., Harms, M. P., Petersen, S. E., Schlaggar, B. L., Corbetta, M., Glasser, M. F., Curtiss, S., Dixit, S., Feldt, C., Nolan, D., Bryant, E., Hartley, T., Footer, O., Bjork, J. M., Poldrack, R., Smith, S., Johansen-Berg, H., Snyder, A. Z., ... WU-Minn HCP Consortium. (2013). Function in the human connectome: task-fMRI and individual differences in behavior. Neurolmage, 80, 169-189. https://doi.org/10.1016/j.neuroimage.2013.05.033

36. Orgs, G., Dovern, A., Hagura, N., Haggard, P., Fink, G. R., \& Weiss, P. H. (2016). Constructing Visual Perception of Body Movement with the Motor Cortex. Cerebral Cortex , 26(1), 440-449. https://doi.org/10.1093/cercor/bhv262

37. Carey, M., Knight, R., \& Preston, C. (2019). Distinct neural response to visual perspective and body size in the extrastriate body area. Behavioural Brain Research, 372, 112063. https://doi.org/10.1016/j.bbr.2019.112063 
38. Zimmermann, M., Mars, R. B., de Lange, F. P., Toni, I., \& Verhagen, L. (2018). Is the extrastriate body area part of the dorsal visuomotor stream? Brain Structure \& Function, 223(1), 31-46. https://doi.org/10.1007/s00429-017-1469-0

39. Bratch, A., Engel, S., Burton, P., \& Kersten, D. (2018). The Fusiform Body Area Represents Spatial Relationships Between Pairs of Body Parts. Journal of Vision, 18(10), 408-408. https://doi.org/10.1167/18.10.408

40. Kanwisher, N., McDermott, J., \& Chun, M. M. (1997). The fusiform face area: a module in human extrastriate cortex specialized for face perception. The Journal of Neuroscience: The Official Journal of the Society for Neuroscience, 17(11), 4302-4311. https://doi.org/10.1523/JNEUROSCI.17-11$\underline{04302.1997}$

41. Puce, A., Allison, T., Bentin, S., Gore, J. C., \& McCarthy, G. (1998). Temporal cortex activation in humans viewing eye and mouth movements. The Journal of Neuroscience: The Official Journal of the Society for Neuroscience, 18(6), 2188-2199. https://www.ncbi.nlm.nih.gov/pubmed/9482803

42. Baseler, H. A., Harris, R. J., Young, A. W., \& Andrews, T. J. (2014). Neural responses to expression and gaze in the posterior superior temporal sulcus interact with facial identity. Cerebral Cortex, 24(3), 737-744. https://doi.org/10.1093/cercor/bhs360

43. Pitcher, D., Pilkington, A., Rauth, L., Baker, C., Kravitz, D. J., \& Ungerleider, L. G. (2020). The Human Posterior Superior Temporal Sulcus Samples Visual Space Differently From Other Face-Selective Regions. Cerebral Cortex, 30(2), 778-785. https://doi.org/10.1093/cercor/bhz125

44. Epstein, R. A., \& Higgins, J. S. (2007). Differential parahippocampal and retrosplenial involvement in three types of visual scene recognition. Cerebral Cortex , 17(7), 1680-1693. https://doi.org/10.1093/cercor/bhl079

45. Sulpizio, V., Committeri, G., \& Galati, G. (2014). Distributed cognitive maps reflecting real distances between places and views in the human brain. Frontiers in Human Neuroscience, 8, 716. https://doi.org/10.3389/fnhum.2014.00716

46. Epstein, R., Graham, K. S., \& Downing, P. E. (2003). Viewpoint-specific scene representations in human parahippocampal cortex. Neuron, 37(5), 865-876. https://doi.org/10.1016/s0896$\underline{6273(03) 00117-x}$

47. Aminoff, E. M., Kveraga, K., \& Bar, M. (2013). The role of the parahippocampal cortex in cognition. Trends in Cognitive Sciences, 17(8), 379-390. https://doi.org/10.1016/j.tics.2013.06.009

48. Kravitz, D. J., Saleem, K. S., Baker, C. I., \& Mishkin, M. (2011). A new neural framework for visuospatial processing. Nature Reviews. Neuroscience, 12(4), 217-230. https://doi.org/10.1038/nrn3008

49. Weiner, K. S., Barnett, M. A., Witthoft, N., Golarai, G., Stigliani, A., Kay, K. N., Gomez, J., Natu, V. S., Amunts, K., Zilles, K., \& Grill-Spector, K. (2018). Defining the most probable location of the parahippocampal place area using cortex-based alignment and cross-validation. Neurolmage, 170, 373-384. https://doi.org/10.1016/j.neuroimage.2017.04.040

50. Vann, S. D., Aggleton, J. P., \& Maguire, E. A. (2009). What does the retrosplenial cortex do? Nature Reviews. Neuroscience, 10(11), 792-804. https://doi.org/10.1038/nrn2733

51. Grill-Spector, K., Kourtzi, Z., \& Kanwisher, N. (2001). The lateral occipital complex and its role in object recognition. Vision Research, 41(10-11), 1409-1422. https://doi.org/10.1016/s00426989(01)00073-6

52. Bar, M. (2004). Visual objects in context. Nature Reviews. Neuroscience, 5(8), 617-629. https://doi.org/10.1038/nrn1476

53. Beauchamp, M. S., Lee, K. E., Haxby, J. V., \& Martin, A. (2002). Parallel visual motion processing streams for manipulable objects and human movements. Neuron. https://www.sciencedirect.com/science/article/pii/S0896627302006426 
54. Kourtzi, Z., \& Kanwisher, N. (2001). Representation of perceived object shape by the human lateral occipital complex. Science, 293(5534), 1506-1509. https://doi.org/10.1126/science.1061133

55. Victoria, L. W., Pyles, J. A., \& Tarr, M. J. (2019). The relative contributions of visual and semantic information in the neural representation of object categories. Brain and Behavior, 9(10), e01373. https://doi.org/10.1002/brb3.1373

56. Logothetis, N. K. (2003). The underpinnings of the BOLD functional magnetic resonance imaging signal. The Journal of Neuroscience: The Official Journal of the Society for Neuroscience, 23(10), 3963-3971. https://www.ncbi.nlm.nih.gov/pubmed/12764080

\section{Methods}

\section{Data acquisition and participants}

Data used in the present study were collected by the Washington University-Minnesota Consortium of the Human Connectome Project (HCP) (Van Essen et al., 2013) as part of the young adult (S1200) release. This included resting- and task-state functional neuroimaging data (see following Methods sections for more details on each). We obtained the minimally preprocessed (Glasser et al., 2013) neuroimaging data for $N=352$ healthy young adults. Following the pipeline outlined in Ito et al. (2020a), this $N=352$ subset of the HCP participants were selected to ensure high quality scan data and no familial relations. All study procedures were approved by the Institutional Review Board of Rutgers University-Newark. Further details on participant recruitment can be found in Van Essen et al. (2013).

We used a split-sample validation approach to minimize false discovery rate (Anderson and Magruder, 2017). The cohort of HCP participants in the present study $(N=352)$ were randomly allocated to either a discovery $(n=176)$ or replication $(n=176)$ dataset. Each dataset was analyzed identically but independently. Participants in the discovery dataset (77 men, 99 women) had the following age ranges: 22-25 years (26.14\%), 26-30 years (41.48\%), 31-35 years (30.68\%), and $36+$ years (1.7\%). Participants in the replication dataset (93 men, 83 women) had the following age ranges: $22-25$ years (22.16\%), 26-30 years (43.18\%), 31-35 years $(34.09 \%)$, and $36+$ years $(0.57 \%)$. Results presented in figures refer to the discovery dataset, with replication dataset results reported in-text.

\section{Task paradigm}

In a subset of analyses, we used data corresponding to seven HCP tasks and 24 task conditions that sampled diverse cognitive domains: emotion, gambling reward, language, motor, relational reasoning, social cognition, and working memory. In all other analyses we focused on the working memory/category-specific representation n-back 
task (see Barch et al., 2013 for more details on all tasks). Prior studies suggest that the nback task can be used to test hypotheses regarding the function(s) of specific brain areas (Drobyshevsky et al., 2006). Further, manipulating how far back a participant must remember (0-back versus 2-back) allows for the assessment of working memory maintenance (along with other contrasts, such as stimulus type). While working memory is not the main interest of the present study, specifically manipulating working memory has the added benefit of promoting task engagement.

The cognitive domain of interest in the present study was the processing of semantic categories (Haxby et al., 2001; Wierenga et al., 2009; Op de Beeck et al., 2019). Category-specific representations were embedded in the n-back task via blocks of trials that presented images of four reliably studied and distinct visual semantic categories (Peelen and Downing, 2005; Osher et al., 2016): (1) bodies (Downing et al., 2001; Taylor et al., 2007); (2) faces (Kanwisher et al., 1997; Kanwisher and Yovel, 2006; lidaka, 2014); (3) places (Park and Chun, 2009; Weiner et al., 2018); and (4) tools (Grill-Spector et al., 2001; Wierenga et al., 2009; Matić et al., 2020).

The specifics of the n-back task are detailed in Barch et al. (2013). Briefly, the nback task included 2 runs with blocks of visual category trials (8 blocks, 10 trials each, 2.5 seconds each) and fixation blocks (4 total, 15 seconds each). Half of the blocks were 0back and half were 2-back. During 0-back working memory tasks, participants determined via button-press whether presented stimuli (images of either bodies, faces, places, or tools) matched a constant target shown in the initial cue screen. During 2-back working memory tasks, participants were asked to indicate if the present stimulus matched a target from two screens back. Given that working memory load was not the main interest of the present study, results presented here reflect an average across 0 back and 2-back conditions (i.e., fMRI data was averaged after all preprocessing steps), except for in analyses examining "response profiles" (i.e., all 24 HCP conditions were assessed separately).

\section{MRI parameters}

All MRI data were collected at Washington University in St. Louis, Missouri, USA in a customized Siemens 3T "Connectome Skyra" scanner. Whole-brain, multiband, and echo-planar images (EPI) were acquired with a 32-channel head coil. The repetition time (TR) was $720 \mathrm{~ms}$; the echo time (TE) was $33.1 \mathrm{~ms}$; the flip angle was 52 degrees; the bandwidth (BW) was $2290 \mathrm{~Hz} / \mathrm{Px}$; in-plane field-of-view (FoV) was 208 x $180 \mathrm{~mm} ; 72$ slices; $2.0 \mathrm{~mm}$ isotropic voxels; and the multiband acceleration factor was 8 . Whole-brain and high-resolution T1-weighted and T2-weighted anatomical scans were also acquired, with an isotropic voxel resolution of $0.7 \mathrm{~mm}$. All imaging data were collected over two 
days for each participant. Each day included two resting-state fMRI scans, each lasting 14.4 minutes (for a total of approximately 29 minutes of rest data per day). During rest, participants were instructed to keep their eyes open and fixated. Task fMRI (30 minutes of task data per day) followed resting-state fMRI scans on each day. Each of the 7 tasks were performed over two consecutive runs. Further details on MRI protocols and parameters can be found in Van Essen et al. (2013).

\section{fMRI preprocessing}

We acquired minimally preprocessed data (Glasser et al., 2013) from the HCP database (www.humanconnectome.org). In-brief, this included: anatomic reconstruction and segmentation; motion correction; intensity normalization; and EPI reconstruction to a standard (surface-based) template; MSM-All registration (multimodal surface matching registration based on areal features from multiple sources, including: cortical folding/sulcal depth, myelin maps, resting-state networks, and visuotopic maps; Glasser et al., 2016). The resulting data were in CIFTI grayordinate (i.e., vertex) space. We performed additional preprocessing steps on cortical vertices $(59,412$ vertices across the two hemispheres). On both resting- and task-state data, we removed the first five frames of each fMRI run, demeaned and detrended the timeseries, and performed nuisance regression. We followed a variant on methods suggested by Ciric et al. (2017) to model and regress out nuisance parameters, including motion and physiological noise. We performed nuisance regression to remove six motion parameters, their derivatives, and quadratics (24 total). Anatomical CompCor (aCompCor; Behzadi et al., 2007) was used on white matter and ventricle timeseries to model physiological noise. The first five principal components from white matter and ventricles were independently extracted. These parameters, their derivatives, and the quadratics of all regressors were removed (40 total). Altogether, 64 nuisance parameters were modeled and associated variance removed from the data.

Global signal was not removed given evidence that it can artificially introduce negative relationships (Murphy et al., 2009; Power et al., 2014) and is non-standard for task activation analyses. However, the nuisance parameters modeled and removed with aCompCor contain components similar to the proposed global signal, with the added benefit of not removing gray matter signals (Power et al., 2018; Ito et al., 2020b).

Task-state activity estimation 
Cortical activations related to task-state fMRI conditions (n-back visual semantic categories) were estimated by a standard general linear model (GLM), which convolved task timing (per block-design condition) with the SPM canonical hemodynamic response function (Friston et al., 1994). This was performed per cortical vertex, each belonging to a parcel (or region) of the Glasser et al. (2016) multimodal parcellation (MMP) atlas. Average coefficients of the GLMs were utilized for each MMP parcel or functional complex (described further in the Methods section below 'Identification of functional complexes') as estimates of visual category evoked brain activity.

\section{Identification of functional complexes}

To address the question of whether distributed predictive models (Cole et al., 2016; Cole et al., 2021) apply to category selective functional responses, we focused on the functional brain regions identified in Osher et al. (2016) given the similarity of the four visual semantic categories in that study to the HCP n-back task studied herein. Additionally, as suggested by Poldrack et al. (2019), variables that will be used in predictive analyses should not be chosen in an empirically-driven manner (e.g., from post hoc cross validation and/or optimization procedures) because this has the potential to artificially inflate prediction accuracy due to circularity. Thus, selecting functional regions of interest a priori has the benefit of controlling false positives in prediction accuracy, particularly with our focus on the well-established visual category literature. Upon further review of the literature as well as the parcels belonging to the Glasser et al. (2016) MMP atlas, we observed that these functional regions: (1) contained multiple MMP parcels (e.g., the parahippocampal place area or PPA, which contained 6 MMP parcels as in Weiner et al., 2018); (2) did not have an exact terminological match in the MMP atlas; and/or (3) included two discrete locations (e.g., the retrosplenial complex, or RSC, often studied in conjunction with the PPA). To address this we first performed a literature search of each of the Osher et al. (2016) functional regions with Google scholar (https://scholar.google.com/) and Neurosynth (https://neurosynth.org/), filtering for relevance, year, and citation count (when possible) (see Table 2 source references). We focused further on publications that had corresponding volumetric coordinates (or MMP surface parcel labels when possible), and generated a list of Montreal Neurological Institute $(\mathrm{MNI}) \mathrm{x} / \mathrm{y} / \mathrm{z}$ volumetric coordinates for each functional region (with a minimum of 10 entries per region). We then used the average $x / y / z$ coordinate set as a reference to identify corresponding MMP parcels (Table 3).

To convert from volumetric space to parcel space, we employed the following steps (using Connectome workbench, FreeSurfer, and AFNI/SUMA commands, per hemisphere): (1) convert the MMP atlas to FreeSurfer's fsaverage space; (2) convert 
fsaverage to the surface-mesh MNI-152 template (which is also included in AFNI); (3) convert surface $\mathrm{MNI}-152$ template to volumetric MNI-template and obtain coordinates. Additional commands were used to ensure that the templates aligned as best as possible, including zero-padding, thicker ribbons (that were later filled), and adding MMP sphere labels. To obtain $x / y / z$ coordinates, we divided the template into equal portions perpendicular to the long axis of a given sphere label, identified the middle division, then calculated the centroid. We then found the MMP parcels that were physically closest to the reference coordinate set for each functional region. We then referred to the Glasser et al. (2016) supplemental results (as well as publications they based the MMP atlas upon; see source references with asterisks in Table 2) to exclude regions that were physically close but functionally irrelevant (e.g., auditory regions near the pSTS, which was a potential ROI for face processing).

The final set of regions, including their proposed category selectivity, network affiliations, and source references are listed in Table 2. The term "functional complex" indicates firstly that more than one MMP parcel comprised each complex, and secondly serves to distinguish an MMP atlas parcel from a category-selective (based on prior literature) complex in the present study. Further, in some cases there were two functionally relevant complexes in the literature (e.g., PPA and RSC, both relevant to place processing, as in Osher et al., 2016), therefore both were included. When appropriate, we applied the Cole-Anticevic brain-wide network partition (CAB-NP; Ji et al., 2019; Fig. 1C), which was based on HCP resting-state fMRI data and was generated from a Louvain community detection algorithm that assigned MMP parcels to 12 functional networks (labeled via distinct colors in Fig. 1C). Table 2 lists the network assignments of each region, which is further reflected in Results figures by color labeling matching Fig. 1C. The reference sets of volumetric coordinates $(x / y / z)$ are listed in Table 3.

\begin{tabular}{|c|c|c|c|c|}
\hline $\begin{array}{c}\text { Visual } \\
\text { category }\end{array}$ & $\begin{array}{c}\text { Parcel } \\
\text { (acronym, } \\
\text { L/R indices) }\end{array}$ & Network & $\begin{array}{c}\text { Functional } \\
\text { complex }\end{array}$ & Source references \\
\hline \multicolumn{5}{|l|}{ Bodies } \\
\hline & $\begin{array}{c}\text { MST, 2, } \\
182\end{array}$ & VIS2 & EBA & $\begin{array}{l}\text { 1. Malikovic et al. (2007). Cereb Cortex, 17(3), 562-574.* } \\
\text { 2. Fischl et al. (2008). Cereb Cortex, 18(8), 1973-1980.* } \\
\text { 3. Kolster et al. (2010). J Neurosci, 30(29), 9801-9820.* } \\
\text { 4. Abdollahi et al. (2014). Neurolmage, 99, 509-524.* } \\
\text { 5. Downing et al. (2001). Science, 293(5539), 2470-2473. } \\
\text { 6. Peelen and Downing (2005). Hum Brain Map, 25(4), 402-408. } \\
\text { 7. Spiridon et al. (2006). Hum Brain Map, 27(1), 77-89. } \\
\text { 8. Taylor et al. (2007). J Neurophysiol, 98(3), 1626-1633. } \\
\text { 9. Vocks et al. (2010). Cogn Affect Behav Neurosci, 10(3), 422-429. }\end{array}$ \\
\hline
\end{tabular}


bioRxiv preprint doi: https://doi.org/10.1101/2022.02.19.481103; this version posted February 22, 2022. The copyright holder for this preprint (which was not certified by peer review) is the author/funder, who has granted bioRxiv a license to display the preprint in perpetuity. It is made available under aCC-BY-NC-ND 4.0 International license.

\begin{tabular}{|c|c|c|c|c|}
\hline & & & & $\begin{array}{l}\text { 10. Shultz et al. (2011). Soc Cogn Affect Neurosci, 6(5), 602-611. } \\
\text { 11. Barch et al. (2013). Neurolmage, 80, 169-189. } \\
\text { 12. Ross et al. (2014). Front Hum Neurosci, 8, 941. } \\
\text { 13. Orgs et al. (2016). Cereb Cortex , 26(1), 440-449. } \\
\text { 14. Zimmermann et al. (2018). Brain Struct Func, 223(1), 31-46. }\end{array}$ \\
\hline & $\begin{array}{c}\mathrm{PH}, 138 \\
318\end{array}$ & VIS2 & EBA & $\begin{array}{l}\text { 1. von Economo and Koskinas (1925). J Springer.* } \\
\text { 2. Triarhou (2007). Stereotac Func Neurosurg, 85(5), 195-203.* } \\
\text { [remaining EBA source references same as first row for EBA grouping] }\end{array}$ \\
\hline & $\begin{array}{c}\text { V4t, 156, } \\
336\end{array}$ & VIS2 & EBA & $\begin{array}{l}\text { 1. Larsson and Heeger (2006). J Neurosci, 26(51), 13128-13142.* } \\
\text { 2. Kolster et al. (2010). J Neurosci, 30(29), 9801-9820.* } \\
\text { 3. Abdollahi et al. (2014). Neurolmage, 99, 509-524.* } \\
\text { [remaining EBA source references same as first row for EBA grouping] }\end{array}$ \\
\hline & $\begin{array}{c}\text { FST, } 157 \\
337\end{array}$ & VIS2 & EBA & $\begin{array}{l}\text { 1. Kolster et al. (2010). J Neurosci, 30(29), 9801-9820.* } \\
\text { 2. Abdollahi et al. (2014). Neurolmage, 99, 509-524.* } \\
\text { [remaining EBA source references same as first row for EBA grouping] }\end{array}$ \\
\hline & $\begin{array}{c}\text { TE2p, 136, } \\
316\end{array}$ & DAN & FBA & $\begin{array}{l}\text { 1. von Economo and Koskinas (1925). J Springer.* } \\
\text { 2. Triarhou (2007). Stereotac Func Neurosurg, 85(5), 195-203.* } \\
\text { 3. Taylor et al. (2007). J of Neurophysiol, 98(3), 1626-1633. } \\
\text { 4. Hodzic et al. (2009). Neurolmage, 45(4), 1264-1271. } \\
\text { 5. Taylor et al. (2010). J of Neurophysiol, 103(3), 1467-1477. } \\
\text { 6. Vocks et al. (2010). Cogn Affect Behav Neurosci, 10(3), 422-429. } \\
\text { 7. Ewbank et al. (2011). J Neurosci, 31(15), 5635-5642. } \\
\text { 8. Hashimoto and Iriki (2013). Euro J Neurosci, 37(11), 1747-1753. } \\
\text { 9. Ross et al. (2014). Front Hum Neurosci, 8, 941. } \\
\text { 10. Kim et al. (2014). Front Hum Neurosci, 8, 632. } \\
\text { 11. Orgs et al. (2016). Cereb Cortex, 26(1), 440-449. } \\
\text { 12. Zimmermann et al. (2018). Brain Struct Func, 223(1), 31-46. } \\
\text { 13. Ross et al. (2020). MethodsX, 7, 100801. }\end{array}$ \\
\hline \multicolumn{5}{|l|}{ Faces } \\
\hline & $\begin{array}{c}\text { FFC, } 18 \\
198\end{array}$ & VIS2 & FFA & $\begin{array}{l}\text { 1. Kanwisher and Yovel (2006). Philos Trans R Soc Lond B Biol Sci, } \\
\text { 361(1476), 2109-2128.* } \\
\text { 2. Glasser and Van Essen (2011). J Neurosci, 31(32), 11597-11616.* } \\
\text { 3. Caspers et al. (2013). Brain Struct Func, 218(2), 511-526.* } \\
\text { 4. Weiner et al. (2014). Neurolmage, 84, 453-465.* } \\
\text { 5. Spiridon et al. (2006). Hum Brain Mapp, 27(1), 77-89. } \\
\text { 6. Peelen and Downing (2005). Hum Brain Mapp, 25(4), 402-408. } \\
\text { 7. Turk-Browne et al. (2010). Front Hum Neurosci, 4, 176. } \\
\text { 8. Nagy et al. (2012). Front Psychol, 3, 141. } \\
\text { 9. Barch et al. (2013). Neurolmage, 80, 169-189. } \\
\text { 10. Kim et al. (2014). Front Hum Neurosci, 8, 632. } \\
\text { 11. Baseler et al. (2014). Cereb Cortex, 24(3), 737-744. } \\
\text { 12. Flack et al. (2015). Cortex, 69, 14-23. } \\
\text { 13. Axelrod and Yovel (2015). PloS One, 10(2), e0117126. } \\
\text { 14. Schobert et al. (2018). Soc Cogn Affect Neurosci, 13(1), 102-113. } \\
\text { 15. Li et al. (2019). Neurosci, 408, 248-258. } \\
\text { 16. Pitcher et al. (2020). Cereb Cortex, 30(2), 778-785. }\end{array}$ \\
\hline & $\begin{array}{c}\text { STSdp, } \\
129,309\end{array}$ & LAN & pSTS & $\begin{array}{l}\text { 1. Engell and Haxby (2007). Neuropsychologia, 45(14), 3234-3241. } \\
\text { 2. von dem Hagen et al. (2011). Cereb Cortex, 21(3), 493-500. } \\
\text { 3. Shultz et al. (2011). Soc Cogn Affect Neurosci, 6(5), 602-611. } \\
\text { 4. Ross et al. (2014). Front Hum Neurosci, 8, 941. } \\
\text { 5. Baseler et al. (2014). Cereb Cortex , 24(3), 737-744. }\end{array}$ \\
\hline
\end{tabular}


bioRxiv preprint doi: https://doi.org/10.1101/2022.02.19.481103; this version posted February 22, 2022. The copyright holder for this preprint (which was not certified by peer review) is the author/funder, who has granted bioRxiv a license to display the preprint in perpetuity. It is made available under aCC-BY-NC-ND 4.0 International license.

\begin{tabular}{|c|c|c|c|c|}
\hline & & & & $\begin{array}{l}\text { 6. Watson et al. (2014). J Neurosci, 34(20), 6813-6821. } \\
\text { 7. Flack et al. (2015). Cortex, 69, 14-23. } \\
\text { 8. Thurman et al. (2016). Neurolmage, 136, 149-161. } \\
\text { 9. Orgs et al. (2016). Cereb Cortex, 26(1), 440-449. } \\
\text { 10. Schobert et al. (2018). Soc Cogn Affect Neurosci, 13(1), 102-113. } \\
\text { 11. Li et al. (2019). Neurosci, 408, 248-258. } \\
\text { 12. Ross et al. (2020). MethodsX, 7, 100801. } \\
\text { 13. Pitcher et al. (2020). Cereb Cortex, 30(2), 778-785. }\end{array}$ \\
\hline & $\begin{array}{c}\text { STSvp, } 130 \\
310\end{array}$ & DMN & pSTS & [remaining pSTS source references same as first row for pSTS grouping] \\
\hline \multicolumn{5}{|l|}{ Places } \\
\hline & $\begin{array}{c}\text { PHA1, 126, } \\
306\end{array}$ & DMN & PPA & $\begin{array}{l}\text { 1. Peelen and Downing (2005). Hum Brain Mapp, 25(4), 402-408. } \\
\text { 2. Spiridon et al. (2006). Hum Brain Mapp, 27(1), 77-89. } \\
\text { 3. Epstein and Higgins (2007). Cereb Cortex, 17(7), 1680-1693. } \\
\text { 4. Henderson (2008). Brain and Cogn, 66(1), 40-49. } \\
\text { 5. Pourtois et al. (2009). Cereb Cortex, 19(8), 1806-1819. } \\
\text { 6. Park and Chun (2009). Neurolmage, 47(4), 1747-1756. } \\
\text { 7. Bastin et al. (2013). J Neurosci, 33(24), 10123-10131. } \\
\text { 8. Barch et al. (2013). Neurolmage, 80, 169-189. } \\
\text { 9. Sulpizio et al. (2014). Front Hum Neurosci, 8, 716. } \\
\text { 10. Marchette et al. (2015). J Neurosci, 35(44), 14896-14908. } \\
\text { 11. Bonner et al. (2016). J of Cogn Neurosci, 28(3), 361-378. } \\
\text { 12. Bellana et al. 2017). Hum Brain Mapp, 38(3), 1155-1171. }\end{array}$ \\
\hline & $\begin{array}{c}\text { PHA2, 155, } \\
335\end{array}$ & DMN & PPA & [remaining PPA source references same as first row for PPA grouping] \\
\hline & $\begin{array}{c}\text { PHA3, 127 } \\
307\end{array}$ & DAN & PPA & [remaining PPA source references same as first row for PPA grouping] \\
\hline & $\begin{array}{c}\text { VMV1, 153 } \\
333\end{array}$ & VIS2 & PPA & $\begin{array}{l}\text { 1. Arcaro et al. (2009). J Neurosci, 29(34), 10638-10652.* } \\
\text { 2. Wang et al. (2015). Cereb Cortex, 25(10), 3911-3931.* } \\
\text { [remaining PPA citations same as first row for PPA grouping] }\end{array}$ \\
\hline & $\begin{array}{c}\text { VMV2, 160 } \\
340\end{array}$ & VIS2 & PPA & $\begin{array}{l}\text { 1. Arcaro et al. (2009). J Neurosci, 29(34), 10638-10652.* } \\
\text { 2. Wang et al. (2015). Cereb Cortex , 25(10), 3911-3931.* } \\
\text { [remaining PPA source references same as first row for PPA grouping] }\end{array}$ \\
\hline & $\begin{array}{c}\text { VMV3, 154, } \\
334\end{array}$ & VIS2 & PPA & $\begin{array}{l}\text { 1. Arcaro et al. (2009). J Neurosci, 29(34), 10638-10652. } \\
\text { 2. Wang et al. (2015). Cereb Cortex , 25(10), 3911-3931. } \\
\text { 3. Wandell and Winawer. (2011). Vision Res, 51(7), 718-737.* } \\
\text { [remaining PPA source references same as first row for PPA grouping] }\end{array}$ \\
\hline & $\begin{array}{c}\text { POS1, 31 } \\
211\end{array}$ & DMN & RSC & $\begin{array}{l}\text { 1. Glasser and Van Essen (2011). J Neurosci, 31(32), 11597-11616.* } \\
\text { 2. Wolbers and Büchel (2005). J Neurosci, 25(13), 3333-3340. } \\
\text { 3. Epstein and Higgins (2007). Cereb Cortex, 17(7), 1680-1693. } \\
\text { 4. Henderson (2008). Brain Cogn, 66(1), 40-49. } \\
\text { 5. Park and Chun (2009). Neurolmage, 47(4), 1747-1756. } \\
\text { 6. Sherrill et al. (2013). J Neurosci, 33(49), 19304-19313. } \\
\text { 7. Sulpizio et al. (2014). Front Hum Neurosci, 8, 716. } \\
\text { 8. Marchette et al. (2015). J Neurosci, 35(44), 14896-14908. } \\
\text { 9. Bellana et al. 2017). Hum Brain Mapp, 38(3), 1155-1171. } \\
\text { 10. } \text { Burles et al. (2017). Brain Struct Func, 222(3), 1481-1493. }\end{array}$ \\
\hline Tools & & & & \\
\hline
\end{tabular}


bioRxiv preprint doi: https://doi.org/10.1101/2022.02.19.481103; this version posted February 22, 2022. The copyright holder for this preprint (which was not certified by peer review) is the author/funder, who has granted bioRxiv a license to display the preprint in perpetuity. It is made available under aCC-BY-NC-ND 4.0 International license.

\begin{tabular}{|c|c|c|c|}
\hline V4, 6, 186 & VIS2 & LOC & $\begin{array}{l}\text { 1. Rottschy et al. (2007). Hum Brain Mapp, 28(10), 1045-1059. } \\
\text { 2. Hansen et al. (2007). J Neurosci, 27(44), 11896-11911.* } \\
\text { 3. Abdollahi et al. (2014). Neurolmage, 99, 509-524.* } \\
\text { 4. Malikovic et al. (2016). Brain Struct Func, 221(4), 1877-1897. } \\
\text { 5. Grill-Spector et al. (1998). Hum Brain Mapp, 6(4), 316-328. } \\
\text { 6. Niemeier et al. (2005). Cereb Cortex, 15(3), 325-331. } \\
\text { 7. Spiridon et al. (2006). Hum Brain Mapp, 27(1), 77-89. } \\
\text { 8. Pourtois et al. (2009). Cereb Cortex, 19(8), 1806-1819. } \\
\text { 9. Silvanto et al. (2010). Euro J Neurosci, 32(1), 165-171. } \\
\text { 10. Cant and Goodale (2011). J Neurosci, 31(22), 8248-8258. } \\
\text { 11. McFadden et al. (2012).. BMC Psychiatry, 12, 213. } \\
\text { 12. Nagy et al. (2012). Front Psychol, 3, 141. } \\
\text { 13. Karanian and Slotnick (2015). Brain Res, 1603, 124-132. } \\
\text { 14. Chiou and Lambon Ralph (2016). J Neurosci 36(17), 4662-4668. } \\
\text { 15. Erdogan et al. (2016). J Cogn Neurosci, 28(6), 869-881. } \\
\text { 16. Chouinard et al. (2017). J Cogn Neurosci, 29(5), 881-895. } \\
\text { 17. Zimmermann et al. (2018). Brain Struct Func, 223(1), 31-46. } \\
\text { 18. Bahram et al. (2020). Berlin, 2(1), s42466-020. }\end{array}$ \\
\hline V8, 7, 187 & VIS2 & LOC & $\begin{array}{l}\text { 1. Hadjikhani et al. (1998). Nat Neurosci, 1(3), 235-241.* } \\
\text { 2. Abdollahi et al. (2014). Neurolmage, } 99,509-524 .^{*} \\
\text { [remaining LOC source references same as first row for LOC grouping] }\end{array}$ \\
\hline $\begin{array}{l}\text { LO1, 20, } \\
200\end{array}$ & VIS2 & LOC & $\begin{array}{l}\text { 1. Larsson and Heeger (2006). J Neurosci, 26(51), 13128-13142.* } \\
\text { 2. Hansen et al. (2007). J Neurosci, 27(44), 11896-11911.* } \\
\text { 3. Abdollahi et al. (2014). Neurolmage, 99, 509-524.* } \\
\text { 4. Malikovic et al. (2016). Brain Struct Func, 221(4), 1877-1897. } \\
\text { [remaining LOC source references same as first row for LOC grouping] }\end{array}$ \\
\hline $\begin{array}{l}\mathrm{LO} 2,21 \\
201\end{array}$ & VIS2 & LOC & $\begin{array}{l}\text { 1. Larsson and Heeger (2006). J Neurosci, 26(51), 13128-13142.* } \\
\text { 2. Hansen et al. (2007). J Neurosci, 27(44), 11896-11911.* } \\
\text { 3. Abdollahi et al. (2014). Neurolmage, 99, 509-524.* } \\
\text { 4. Malikovic et al. (2016). Brain Struct Func, 221(4), 1877-1897. } \\
\text { [remaining LOC source references same as first row for LOC grouping] }\end{array}$ \\
\hline PIT, 22, 202 & VIS2 & LOC & $\begin{array}{l}\text { 1. Kanwisher and Yovel (2006). Philos Trans R Soc Lond B Biol Sci, } \\
\text { 361(1476), 2109-2128.* } \\
\text { 2. Tsao et al. (2008). Proc Natl Acad Sci USA, 105(49), 19514-19519.* } \\
\text { 3. Kolster et al. (2010). J Neurosci, 30(29), 9801-9820.* } \\
\text { 4. Abdollahi et al. (2014). Neurolmage, 99, 509-524.* } \\
\text { 5. Malikovic et al. (2016). Brain Struct Func, 221(4), 1877-1897. } \\
\text { [remaining LOC source references same as first row for LOC grouping] }\end{array}$ \\
\hline $\begin{array}{c}\text { V3CD, 158, } \\
338\end{array}$ & VIS2 & LOC & $\begin{array}{l}\text { 1. Abdollahi et al. (2014). Neurolmage, } 99,509-524 .^{*} \\
\text { 2. Malikovic et al. (2016). Brain Struct Func, } 221(4), 1877-1897 . \\
\text { [remaining LOC source references same as first row for LOC grouping] }\end{array}$ \\
\hline
\end{tabular}

(Column 1) Visual categories to group remaining rows into four categories: bodies, faces, places, and tools. (Column 2) The MMP (Glasser et al., 2016) parcel (brain region) acronym and corresponding indices (numbers in the MMP atlas) for the left and right (L/R) hemispheres. MST = medial superior temporal area; $\mathrm{PH}=$ basal parietal area (superior); V4t $=$ visual area V4t; FST $=$ fundus of the superior temporal area; TE2 $p=$ temporal area 2 (posterior); FFC $=$ fusiform face complex (MMP terminology for this parcel); STSdp = posterior superior temporal sulcus (dorsal); STSvp = posterior superior temporal sulcus (ventral); PHA1-3 = parahippocampal areas 1-3; VMV1-3 = ventromedial visual areas 1-3; POS1 = parieto-occipital sulcus area $1 ; \mathrm{V} 4=$ fourth visual area; V8 = eighth visual area; LO1 = lateral occipital area 1 ; LO2 = lateral occipital area 2; PIT = posterior inferotemporal; V3CD = area V3CD. (Column 3) CAB-NP (Ji et al., 2019) network each parcel belongs to (as in Fig. 2) (Column 4) The functional complex that each parcel belongs to, based upon prior literature. EBA = extrastriate body area; FBA = fusiform body area; FFA = fusiform face area (we used "FFA" versus "FFC" for consistency with the broader literature); PSTS = posterior superior temporal sulcus; PPA = 
parahippocampal place area; RSC = retrosplenial complex; $L O C=$ lateral occipital complex. (Column 5) Source references (with standard National Library of Medicine journal abbreviations) used to identify volumetric coordinates (Table 3) corresponding to each functional complex and/or source references to corroborate MMP parcels (identified with asterisks) given their reference in Glasser et al., 2016.

Table 3. Average MNI coordinates for each functional complex from the literature.

\begin{tabular}{|c|c|c|c|c|c|c|}
\hline & \multicolumn{5}{|c|}{ Left hemisphere } & \multicolumn{3}{c|}{ Right hemisphere } \\
\hline $\begin{array}{c}\text { Functional } \\
\text { Complex }\end{array}$ & $\begin{array}{c}\text { x } \\
\text { mean (SD) }\end{array}$ & $\begin{array}{c}\mathbf{y} \text { mean (SD) } \\
\text { mean (SD) }\end{array}$ & $\begin{array}{c}\text { x } \\
\text { mean (SD) }\end{array}$ & $\begin{array}{c}\text { y } \\
\text { mean (SD) }\end{array}$ & mean (SD) \\
\hline EBA & $-48.9(6.6)$ & $-73(4.6)$ & $2.7(8.3)$ & $45.7(6.5)$ & $-69.5(3.5)$ & $-0.7(3.9)$ \\
\hline FBA & $-39.7(4)$ & $-44.2(8.2)$ & $-20.2(1.6)$ & $41.9(3.5)$ & $-47(6.2)$ & $-21.5(3.7)$ \\
\hline FFA & $-41.2(3.6)$ & $-53.6(7.2)$ & $-18.6(3.9)$ & $40.6(3.5)$ & $-51.7(3.6)$ & $-18.6(5.2)$ \\
\hline PSTS & $-51.8(6.5)$ & $-49.9(9.9)$ & $8(6)$ & $51.3(4.1)$ & $-49(8.1)$ & $8.6(4.4)$ \\
\hline PPA & $-27.2(4.9)$ & $-44.6(6.2)$ & $-9(4.7)$ & $25.6(4.4)$ & $-42.4(5.5)$ & $-10.3(5.1)$ \\
\hline RSC & $-13.3(4.2)$ & $-56.5(6)$ & $10(6.2)$ & $14.3(5)$ & $-54(5.6)$ & $9.3(7.8)$ \\
\hline LOC & $-45.7(7.1)$ & $-70.4(8.5)$ & $-8.5(6.3)$ & $42.5(6.4)$ & $-69.4(11.1)$ & $-9(7.2)$ \\
\hline
\end{tabular}

(Column 1) The functional complexes assessed in the present study. Naming conventions are the same as in Table 2. (Columns 2-4) Stereotactic ( $x / y / z)$ MNI coordinates for the left hemisphere; mean and standard deviation (SD) of at least 10 publications per complex (see Table 2 for full list of source references). Publications that listed coordinates in Talairach space were converted to MNI space following Lacadie et al., (2008). Note that some publications reported multiple functional localizer experiments, which were treated as separate entries in obtaining average coordinates. (Columns 5-7) The same as in columns 2-4, but for the right hemisphere. These coordinate sets were used as references to locate corresponding MMP (Glasser et al., 2016) parcels, yielding surface based functional complexes.

\section{Resting-state functional connectivity estimation}

Functional connectivity (FC) was modeled as a series of target dependent variables (i.e., each parcel or region), each with multiple source independent variables (i.e., other parcels or regions) (Fig. 7C, step 1). As in Cole et al. (2016), source vertices excluded all vertices within a $10 \mathrm{~mm}$ dilated mask of the target parcel (as well as excluding vertices of the target parcel itself). This reduced potential circularity related to spatial autocorrelation by avoiding nearby vertices in predicting the activity of the target. Modified source sets of vertex-level timeseries were then averaged together based on their Glasser et al. (2016) MMP parcel assignment. FC was then estimated with a method proposed by Sanchez-Romero and Cole (2021) termed combinedFC. CombinedFC integrates the standard methods of bivariate correlation, partial correlation, and multiple regression together to account for causal confounds better than each method alone (Fig. 
1A). Briefly, each participant's resting-state data (pre-processed) was firstly assessed with partial correlation (amongst each pair of regions, conditioning on all other regions) to find conditional associations, which were examined for statistical significance. This step addressed potential confounders and causal chains (Spirtes et al. 2000), and built an initial connectivity architecture by adding in significantly correlated edges only. Then, bivariate correlation estimates (i.e., Pearson correlation) for each pair of connected regions (from the initial partial correlation step) were used to remove spurious edges resulting from conditioning on colliders (specifically, edges where r was statistically equal to zero). Lastly, a multiple regression procedure was performed to scale the estimated edge weights. For each target region functioning as a response variable, all other connected source regions functioned as regressors to estimate the final combinedFC weight. See Sanchez-Romero and Cole (2021) for further detail.

\section{Activity flow generative model}

Activity flow modeling (or "mapping") (Fig. 7) is a generative, mechanistic modeling method that predicts brain activity based upon the inherent link between activation patterns and functional connectivity (Norman-Haignere et al., 2012; Hermundstad et al., 2013; Cole et al., 2014; Ito et al., 2020a). Activity flow was developed by Cole et al. (2016) (see here for further details: Cocuzza et al., 2022), and is described by the following formula:

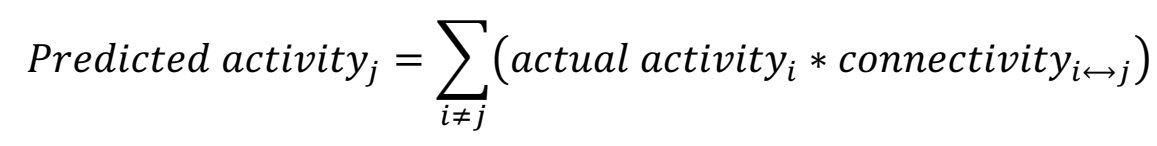

Where $j$ is a held out brain region, and $i$ indexes all other regions in a given parcellation. This method assumes that brain activations are propagated via distributed interactions. Applied to research questions on functional processing, activity flow predicts taskevoked (functionally relevant) activity in held-out target regions as the sum of the taskevoked activity in all other source regions scaled by FC estimates between the target region and corresponding source regions. Using a simulated model of neural processing, Cole et al. (2016) found that activity flow mapping exhibited the highest prediction accuracies when a global coupling parameter was high and the strength of recurrent (local) connections was low (Fig. 7B). Critically, this suggests that the successful application of activity flow mapping to empirical brain data reflects similar high global coupling properties being preserved in real (rather than simulated) data. Thus, the success of activity flow mapping can be taken as a proxy for how much distributed signals 
bioRxiv preprint doi: https://doi.org/10.1101/2022 02 19.481103; this version posted February 22, 2022. The copyright holder for this preprint (which was not certified by peer review) is the author/funder, who has granted bioRxiv a license to display the preprint in perpetuity. It is made available under aCC-BY-NC-ND 4.0 International license.

contribute to a given activation (see Methods section on Category selectivity for more details).

A activity flow mapping

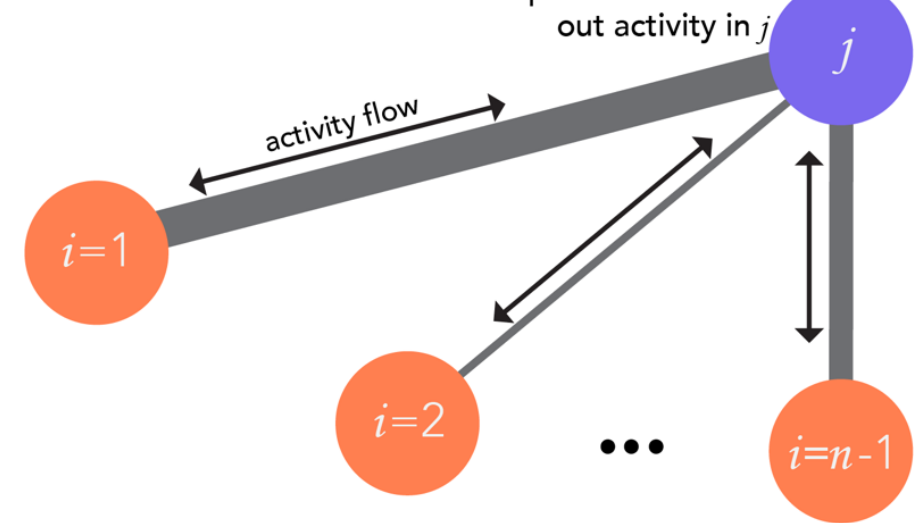

j's predicted activity $=\sum_{i \neq j}\left(i^{\prime}\right.$ s actual activity $\mathbf{X}$ connectivity $\left.i \leftrightarrow j\right)$

C activity flow mapping example

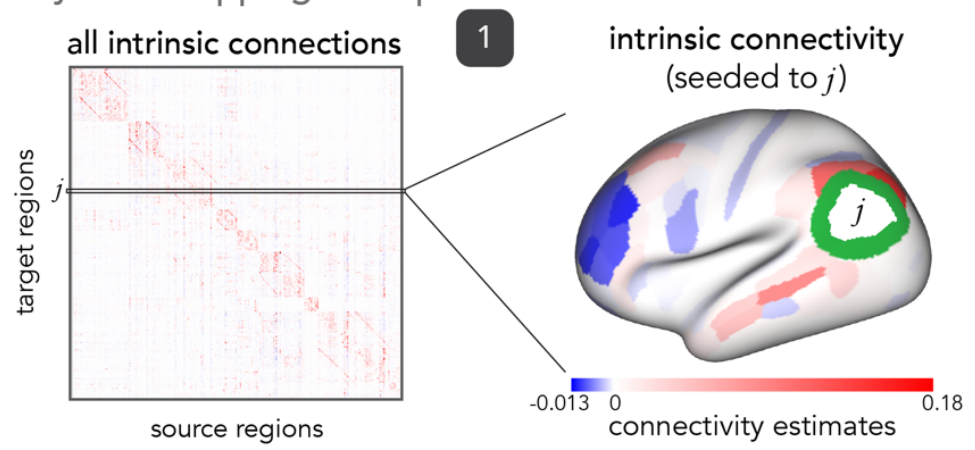

all predicted task activations

\section{6}
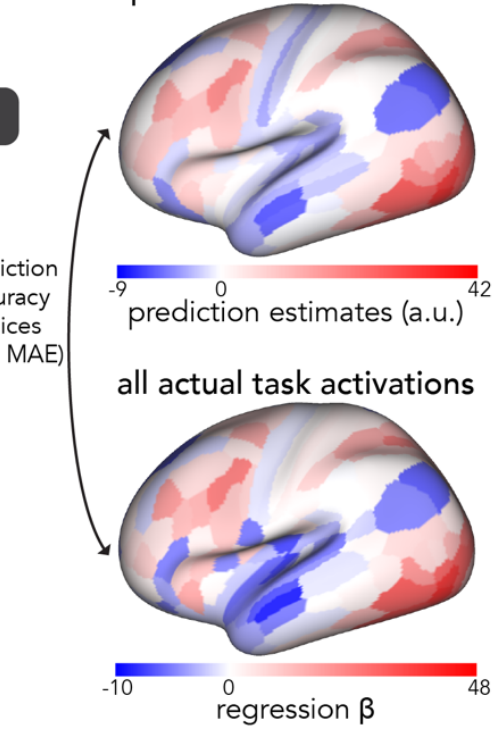

B activity flow mapping simulations

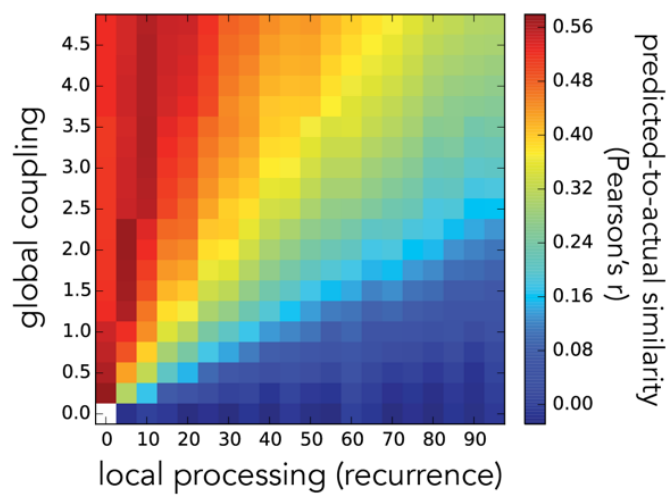

2 actual task activations ( $j$ held out)

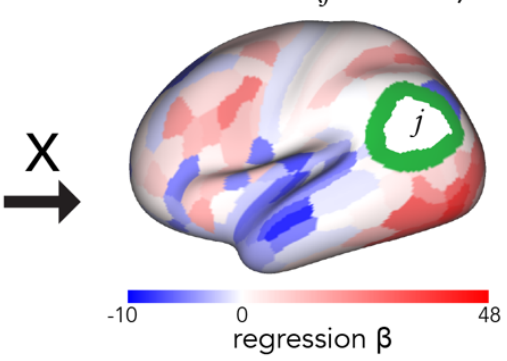

3

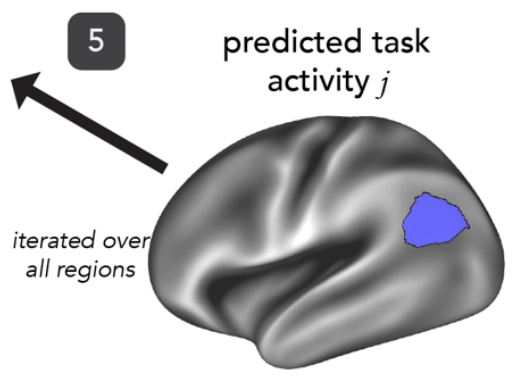

4 activity flow map (contributions to $j$ )

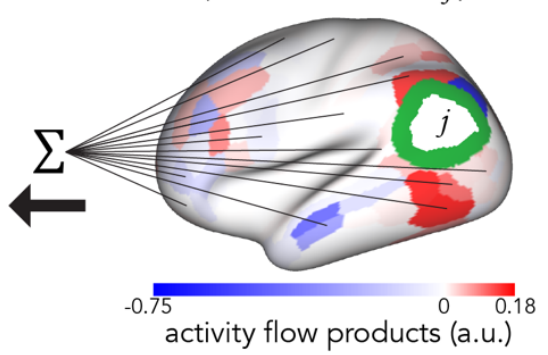

$j$ to-be-predicted region (held out)

excluded from analysis 
Figure 7. Activity flow mapping procedure to predict task activity in held out brain regions. (A) Activity flow mapping toy diagram and formula. Task activity for held-out region $j$ (purple) is predicted by the sum of task activity of all other cortical regions, $i$ (coral) ( $n=$ total number of regions), weighted by their connectivity estimates with $j$ (gray). (B) Activity flow simulation results (reproduced with permission from Cole et al., 2016) demonstrating that activity flow mapping is most successful when distributed processing mechanisms are high and local processing mechanisms are low. (C) Example of activity flow mapping with empirical data (steps 1-6) (reproduced with permission from Cocuzza et al., 2022). For a given target region $j$, estimates of intrinsic (e.g., resting-state) connectivity between $j$ and all other source regions (step 1) are multiplied by all other regions' actual task activations (step 2). This yields an activity flows map quantifying the contribution of all other regions' activity flow upon the held-out region, $j$, for the task of interest (step 3). These are summed to equal the predicted task activity of $j$ (step 4). This procedure is iterated over all regions to generate a whole-cortex map of activity-flow predicted task activations (step 5). This is compared with the actual whole-cortex map of task activations via Pearson's $r, M A E$, and $R_{2}$ to estimate prediction accuracy (step 6). Importantly, this modeling scheme is flexible to different estimates of connectivity. Source vertices not included in the analyses $(10 \mathrm{~mm}$ from the target region $j$; Methods) are depicted in green.

To model category-selective responses to bodies, faces, places, and tools we applied the activity flow predictive model to each of these categories independently. Actual task activations used were beta estimates generated by task GLMs (described in the Methods section above, Task-state activity estimation), with a focus on the MMP regions comprising functional complexes as the held out target regions (described in the Methods section above, Identification of functional complexes). Functional connectivity estimates between targets (functional complex regions) and sources (all other connected regions) were based upon resting-state timeseries and estimated via combinedFC (described in the Methods section above, Resting-state functional connectivity). To assess prediction accuracy, activity-flow-predicted category responses were compared to actual (i.e., empirical) category responses via Pearson r correlation coefficient (NumPy corrcoef function in Python), mean absolute error (MAE), and the coefficient of determination $\left(R^{2}\right)$ (sklearn r2_score function in Python).

Each of these indices characterized a different element of prediction accuracy, altogether providing a thorough evaluation of activity flow modeling. In brief, Pearson's $r$ estimated the linear correlation between actual and predicted brain activations, with a potential range from -1 to 1 and the property of scale invariance. MAE measured the arithmetic mean of absolute errors between actual and predicted brain activations, with a sensitivity to scale. The coefficient of determination $\left(R^{2}\right)$ estimated the percent of unscaled variance in the actual brain activations that was accounted for in the predicted activations. Note that $\mathrm{R}^{2}$ maintains a range of negative infinity to 1 ; from 0 to $1 \mathrm{R}^{2}$ can be interpreted as 0 to $100 \%$ variance, but negative values indicate incorrectly scaled predictions. Thus, each index had clear advantages and disadvantages, but altogether provided a complementary, full characterization of prediction accuracy. In all cases, these 
comparison statistics were taken per participant, then averaged across participants to provide a random effects estimate.

\section{Category selectivity}

To assess how selective each functional complex of interest was for a given visual category, we measured the ratio of category activation amplitude divided by noncategory activation amplitude, for both activity-flow-predicted data and actual data, per participant. For example, for the functional complex of the extrastriate body area (EBA) and fusiform body area (FBA) (see Methods above on Identification of functional complexes), we quantified body activations divided by non-body activations. Per participant and functional complex, we used the following formula:

$$
\text { category selectivity }_{\text {complex }}=\frac{\text { mean }(\text { category responses })}{\text { mean(noncategory responses })}
$$

We will henceforth refer to this ratio as category selectivity, which can be interpreted as how many times larger category responses were than non-category responses. For example, if a given participant's body-category selectivity score (for the EBA and FBA complex) was 1.5, then their activations to body images were 1.5 times larger than their activations to non-body images. A category selectivity score of 1.0 reflected the hypothesized null mean given that it quantifies category activations as equal to noncategory activations. The main advantage of utilizing a ratio versus a difference score is that it is less sensitive to beta value scale differences across brain regions.

Prior to calculating the category selectivity score it was necessary to normalize the range of activation values. This was necessary for two reasons: (1) avoiding negative numbers (which would limit interpretability of the selectivity values) and (2) rescaling activation values such that every participant's data was in the same range (which allows for group level comparisons). We used a standard normalization procedure termed minmax normalization (also termed feature scaling). We used the following formula:

$$
x_{\text {normalized }}=a+\frac{(x-\min (x))(b-a)}{(\max (x)-\min (x))}
$$

Where $a$ and $b$ are the upper and lower bounds of the rescaled data, respectively. We used $a=0$ and $b=1.0$; selected to increase interpretability of the category selectivity ratio since all ratios were positive and included a possible minimum of 0 . The minimum and maximum values in the above formula were calculated based on the actual activations 
of a given region set (e.g., EBA and FBA complex for body-category selectivity) across all 24 task conditions $(x)$ for each participant separately. Note that results were highly similar if minimum and maximum values were calculated separately for predicted and actual activations. We additionally identified and removed outlier participants (in category selectivity) following the methods described by Leys et al. (2013) (median absolute deviation), using a highly conservative threshold of $-/+5$ deviations relative to the median. Given this high threshold, outlier removal excluded a small number of participants with category selectivity scores that were not representative of the group average, which is how category selectivity was reported (which had a large sample size of $n=176$ per discovery and replication datasets). Note that statistical significance (see Methods on Experimental design and statistical analysis) was not impacted by removal of outlier participants.

To estimate the contribution of distributed processes to a given categoryselective response, we computed the percent of actual category selectivity (distributed plus local processes) captured by activity-flow-predicted category selectivity (a model based upon distributed processes only, Fig. 7B). We propose that the percent leftover (i.e., not captured via activity flow modeling) is due to either local processes (not included in the model) or any other source of model prediction error (e.g., noise in the data). We used the following formula:

$$
\% \text { contribution of distributed processes }=\left(\frac{\text { predicted category selectivity }}{\text { actual category selectivity }}\right) * 100
$$

If this percentage is statistically greater than $50 \%$, we infer that category selectivity in that functional complex was generated primarily via distributed processing mechanisms. Note that these estimates reflect a lower bound on the contribution of distributed processes, since they can be driven lower not only by local processes but by any source of model prediction error.

\section{Network analyses}

We tested the contributions of each CAB-NP network (Ji et al., 2019; Fig. 1B) to activity flow predictions. We did this at both the levels of: (1) predicted category-specific response, and (2) predicted response profile.

Category-specific network contributions 
The activity flow map (Fig. 7C, step 3; activity flow products, or just "activity flows") quantified how much activity of a given source region was weighted by functional connectivity with the held out target region. We utilized the network-based averages of these "flow" values for each functional complex and its respective visual processing category to probe category-specific network contributions to the activity flow map. Source regions were averaged together based upon their CAB-NP assignments. These contributions were each statistically compared to one another (see Methods below on Experimental design and statistical analysis) to discover the networks that contributed the most to a given activity flow map.

\section{Response profile network contributions}

To measure the network contributions to activity flow predictions at the response profile level (i.e., across all $24 \mathrm{HCP}$ conditions), we conducted a dominance analysis (Azen and Budescu, 2003). We first re-computed the activity-flow predictions (across all conditions for a given functional complex) with source regions iteratively restricted by CAB-NP network assignment. Note that these values exactly summed to the whole-cortex, activity-flow-modeled predictions, thus this approach simply parsed those predictions into their network components. Then, we conducted multiple linear regression (per participant, across conditions), where network-level predicted activations were the predictor variables (12 in total) and actual activity was the response variable. Following dominance analysis, this yielded the "full model" explained variance $\left(R^{2}\right)$, which in the present study refers to the proportion of variance in the actual response profile explained by the network-based, predicted response profile. An iterative subset of multiple regression models were then performed, with all combinations of predictors tested. These can be thought of as subset models because they were iterated on choosing one through eleven predictors, and performing multiple regression on all combinations therein (procedural details are openly available, and can be found here: github.com/dominance-analysis/dominance-analysis). This followed a standard combination calculation, $\mathrm{nCr}$, where $\mathrm{n}$ was the full number of predictors, or 12 , and $\mathrm{r}$ was iterated between one and eleven (note that $r=12$ describes the full model). In the present study, there were a total of 4095 subsets of models for each functional complex assessed. The incremental contribution of each predictor to $R^{2}$ was quantified for each of these subsets of models. Then, the cross-model average of these incremental contributions was taken as the partial $R^{2}$ of each network, which added up to equal the full model $R^{2}$. Given this property of dominance analysis (that the partial $R^{2}$ values add up to the full $R^{2}$ ), the percentage of relative importance of each network was calculated as the partial $R^{2}$ divided by the full $R^{2}$, times 100 . In addition to a straightforward interpretation, the 
dominance analysis approach has the benefit of being robust to multicollinear predictor variables (Kraha et al., 2012).

\section{Experimental design and statistical analysis}

Each functional complex of brain regions that was proposed to be highly responsive to the visual categories of bodies, faces, places, and tools (described in the Methods section above, Identification of functional complexes) had the following estimates: actual activity and activity-flow-predicted activity. For each of these estimates and each visual category, we contrasted category responses to non-category responses (e.g., body vs non-body), hypothesizing that a functional complex would exhibit higher activity (in actual and predicted data) to the category vs the non-category. To test these hypotheses we employed one-tailed, paired samples t-tests on cross-participant data. For each category of interest, non-category activity was the average of the three remaining categories. For example, if body was the category of interest, responses to faces, places, and tools were averaged for non-body activations. This provided a consistent contrast across all four sets of analyses (i.e., always category vs non-category). In each of these sets of analyses, we corrected for false discovery rate (FDR) following Nichols and Hayasaka (2003) in estimates of statistical significance. The p-values reported in the Results section contrasting category vs non-category responses reflect FDR adjusted $p$ values, following the resampling procedure described by Yekutieli and Benjamini (1999). To assess the prediction accuracy of the activity flow modeled results, we compared the actual and activity-flow-predicted activations in each of the four visual category analyses with Pearson's r, MAE, and $\mathrm{R}^{2}$ (Fig. 1). Lastly, we implemented a Max-T nonparametric permutation approach (shuffling conditions over 100,000 permutations) to correct for multiple comparisons when testing whether category selectivity scores revealed for each functional complex were greater than 1.0 (the null mean), as well as testing whether estimates of distributed processing contributions were greater than $50 \%$ (see Methods section on Category selectivity for more on these measures). We likewise used a Max-T nonparametric permutation approach (here with 10,000 permutations due to computational limits) to address multiple comparisons in the network-based analyses (i.e., comparing each network-level result to every other network-level result). These Max- $T$ analyses derived a 95\% confidence interval from the maximum $T$ distribution (Nichols and Holmes, 2002). The max-T procedure was used in any analysis where we could not assume a normal distribution a priori. Throughout pertinent Results sections we refer to the statistical threshold derived from the permuted null models as the max$T$ threshold (d.f.), wherein a statistically significant $p$ value indicates that the real t-statistic was larger than this threshold. Note that for category selectivity and percent distributed 
processing analyses, degrees of freedom were based on sample size with outlier participants removed (see Methods section on Category selectivity). All findings are reported for cortical regions in the right hemisphere for the discovery dataset. Left hemisphere and replication dataset results are reported in the Supplement.

\section{Control analyses}

To assess how critical each functional complex's unique connectivity fingerprint (as in Passingham et al., 2002; Mars et al., 2018) was to respective visual category selectivity results, we performed an FC substitution experiment. For each target functional complex (and per participant), we substituted each complex's connectivity fingerprint with each other functional complex's fingerprint, then recomputed category selectivity (see prior Methods section, Category selectivity). This meant that for each of the four original, or true, prediction models (bodies, faces, places, and tools), there were three null model comparisons. For example: original body complex rsFC substituted with (1) face complex rsFC, (2) place complex rsFC, and (3) tool complex rsFC (repeated likewise for each original model). To preserve an essential property of the connectivity graphs used in the present study, we set self-connections to zero (e.g., right EBA/FBA target to right EBA/FBA source) (for more on constraint considerations for network null models, see: Wiedermann et al., 2016; Markello and Misic, 2020). Additionally, to account for the zero FC weights that were in the null functional complex's self-connection locations, we set connectivity estimates between the original target complex and the null model complex with the transposed estimate, per hemisphere (e.g., right EBA/FBA target to right FFA/pSTS source set with right FFA/pSTS target to right EBA/FBA source). We performed paired samples t-testing to test the alternative hypothesis that the true model predicted category selectivity was greater than the substituted null model predicted category selectivity (for each of the four visual categories and in each of the three respective FC substitution null models)

\section{Code, software, and data accessibility}

All code and software are publicly available on GitHub. The master repository for the present study can be found here: https://github.com/ColeLab/ActFlowCategories. The Activity Flow Toolbox and related software (Cole et al. 2016; Cocuzza et al., 2022) can be found here: https://github.com/ColeLab/ActflowToolbox. Further, the HCP maintains open access imaging data at various levels of processing here: https://www.humanconnectome.org/. Data at other levels of processing or analysis pertinent to the present study are available upon request. 


\section{References (Methods)}

56. Glasser, M. F., Sotiropoulos, S. N., Wilson, J. A., Coalson, T. S., Fischl, B., Andersson, J. L., Xu, J., Jbabdi, S., Webster, M., Polimeni, J. R., Van Essen, D. C., Jenkinson, M., \& WU-Minn HCP Consortium. (2013). The minimal preprocessing pipelines for the Human Connectome Project. Neurolmage, 80, 105-124. https://doi.org/10.1016/j.neuroimage.2013.04.127

57. Ito, T., Brincat, S. L., Siegel, M., Mill, R. D., He, B. J., Miller, E. K., Rotstein, H. G., \& Cole, M. W. (2020b). Task-evoked activity quenches neural correlations and variability across cortical areas. PLoS Computational Biology, 16(8), e1007983. https://doi.org/10.1371/journal.pcbi.1007983

58. Anderson, M. L., \& Magruder, J. (2017). Split-Sample Strategies for Avoiding False Discoveries (No. 23544). National Bureau of Economic Research. https://doi.org/10.3386/w23544

59. Drobyshevsky, A., Baumann, S. B., \& Schneider, W. (2006). A rapid fMRI task battery for mapping of visual, motor, cognitive, and emotional function. Neurolmage, 31(2), 732-744.

https://doi.org/10.1016/j.neuroimage.2005.12.016

60. lidaka, T. (2014). Role of the fusiform gyrus and superior temporal sulcus in face perception and recognition: An empirical review: Neuroimaging of face recognition. The Japanese Psychological Research, 56(1), 33-45. https://doi.org/10.1111/jpr.12018

61. Matić, K., Op de Beeck, H., \& Bracci, S. (2020). It's not all about looks: The role of object shape in parietal representations of manual tools. Cortex; a Journal Devoted to the Study of the Nervous System and Behavior. https://doi.org/10.1016/j.cortex.2020.09.016

62. Glasser, M. F., Coalson, T. S., Robinson, E. C., Hacker, C. D., Harwell, J., Yacoub, E., Ugurbil, K., Andersson, J., Beckmann, C. F., Jenkinson, M., Smith, S. M., \& Van Essen, D. C. (2016). A multimodal parcellation of human cerebral cortex. Nature, 536(7615), 171-178.

https://doi.org/10.1038/nature18933

63. Ciric, R., Wolf, D. H., Power, J. D., Roalf, D. R., Baum, G. L., Ruparel, K., Shinohara, R. T., Elliott, M. A., Eickhoff, S. B., Davatzikos, C., Gur, R. C., Gur, R. E., Bassett, D. S., \& Satterthwaite, T. D. (2017). Benchmarking of participant-level confound regression strategies for the control of motion artifact in studies of functional connectivity. Neurolmage, 154, 174-187.

https://doi.org/10.1016/j.neuroimage.2017.03.020

64. Behzadi, Y., Restom, K., Liau, J., \& Liu, T. T. (2007). A component based noise correction method (CompCor) for BOLD and perfusion based fMRI. Neurolmage, 37(1), 90-101. https://doi.org/10.1016/j.neuroimage.2007.04.042

65. Murphy, K., Birn, R. M., Handwerker, D. A., Jones, T. B., \& Bandettini, P. A. (2009). The impact of global signal regression on resting state correlations: are anti-correlated networks introduced? Neurolmage, 44(3), 893-905. https://doi.org/10.1016/j.neuroimage.2008.09.036

66. Power, J. D., Mitra, A., Laumann, T. O., Snyder, A. Z., Schlaggar, B. L., \& Petersen, S. E. (2014). Methods to detect, characterize, and remove motion artifact in resting state fMRI. Neurolmage, 84, 320-341. https://doi.org/10.1016/j.neuroimage.2013.08.048

67. Power, J. D., Plitt, M., Gotts, S. J., Kundu, P., Voon, V., Bandettini, P. A., \& Martin, A. (2018). Ridding $\mathrm{fMRI}$ data of motion-related influences: Removal of signals with distinct spatial and physical bases in multiecho data. Proceedings of the National Academy of Sciences of the United States of America, 115(9), E2105-E2114. https://doi.org/10.1073/pnas.1720985115

68. Friston, K. J., Holmes, A. P., Worsley, K. J., Poline, J. P., Frith, C. D., \& Frackowiak, R. S. J. (1994). Statistical parametric maps in functional imaging: A general linear approach. Human Brain Mapping, 2(4), 189-210. https://doi.org/10.1002/hbm.460020402 
69. Poldrack, R. A., Huckins, G., \& Varoquaux, G. (2019). Establishment of Best Practices for Evidence for Prediction: A Review. JAMA Psychiatry . https://doi.org/10.1001/jamapsychiatry.2019.3671

70. Lacadie, C. M., Fulbright, R. K., Rajeevan, N., Constable, R. T., \& Papademetris, X. (2008). More accurate Talairach coordinates for neuroimaging using non-linear registration. Neurolmage, 42(2), 717-725. https://doi.org/10.1016/j.neuroimage.2008.04.240

71. Spirtes, P., Glymour, C. N., Scheines, R., \& Heckerman, D. (2000). Causation, Prediction, and Search. MIT Press.

72. Hermundstad, A. M., Bassett, D. S., Brown, K. S., Aminoff, E. M., Clewett, D., Freeman, S., Frithsen, A., Johnson, A., Tipper, C. M., Miller, M. B., Grafton, S. T., \& Carlson, J. M. (2013). Structural foundations of resting-state and task-based functional connectivity in the human brain. Proceedings of the National Academy of Sciences of the United States of America, 110(15), 6169-6174. https://doi.org/10.1073/pnas.1219562110

73. Cole, M. W., Bassett, D. S., Power, J. D., Braver, T. S., \& Petersen, S. E. (2014). Intrinsic and taskevoked network architectures of the human brain. Neuron, 83(1), 238-251. https://doi.org/10.1016/j.neuron.2014.05.014

74. Leys, C., Ley, C., Klein, O., Bernard, P., \& Licata, L. (2013). Detecting outliers: Do not use standard deviation around the mean, use absolute deviation around the median. Journal of Experimental Social Psychology, 49(4), 764-766. https://doi.org/10.1016/j.jesp.2013.03.013

75. Azen, R., \& Budescu, D. V. (2003). The dominance analysis approach for comparing predictors in multiple regression. Psychological Methods, 8(2), 129-148. https://doi.org/10.1037/1082989x.8.2.129

76. Kraha, A., Turner, H., Nimon, K., Zientek, L. R., \& Henson, R. K. (2012). Tools to support interpreting multiple regression in the face of multicollinearity. Frontiers in Psychology, 3, 44. https://doi.org/10.3389/fpsyg.2012.00044

77. Nichols, T., \& Hayasaka, S. (2003). Controlling the familywise error rate in functional neuroimaging: a comparative review. Statistical Methods in Medical Research, 12(5), 419-446. https://doi.org/10.1191/0962280203sm341ra

78. Yekutieli, D., \& Benjamini, Y. (1999). Resampling-based false discovery rate controlling multiple test procedures for correlated test statistics. Journal of Statistical Planning and Inference. https://www.sciencedirect.com/science/article/pii/S0378375899000415

79. Nichols, T. E., \& Holmes, A. P. (2002). Nonparametric permutation tests for functional neuroimaging: a primer with examples. Human Brain Mapping, 15(1), 1-25. https://doi.org/10.1002/hbm.1058

80. Wiedermann, M., Donges, J. F., Kurths, J., \& Donner, R. V. (2016). Spatial network surrogates for disentangling complex system structure from spatial embedding of nodes. Physical Review. E, 93, 042308. https://doi.org/10.1103/PhysRevE.93.042308

81. Markello, R. D., \& Misic, B. (2021). Comparing spatial null models for brain maps. Neurolmage, 236, 118052. https://doi.org/10.1016/j.neuroimage.2021.118052 


\section{A}

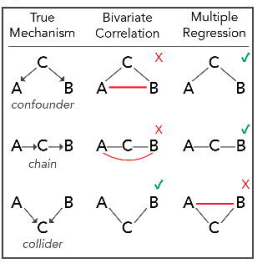

B

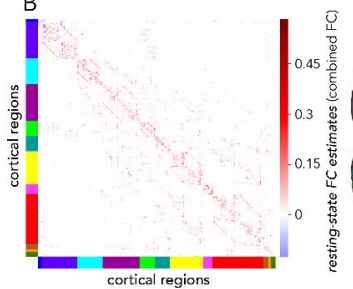

$C$

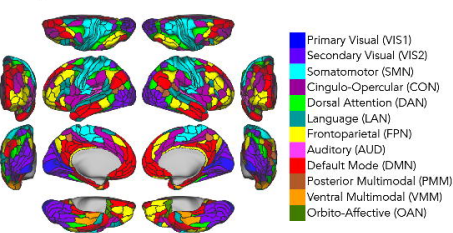

\section{predicted}

$$
r=0.92, R^{2}=0.80
$$

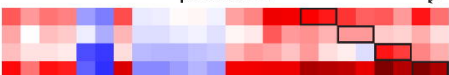

rEBA \& rFBA rFFA \& roSTS rPPA \& rRSC $\mathrm{rLOC}$

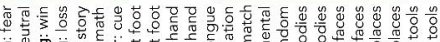

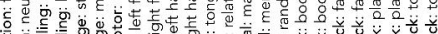

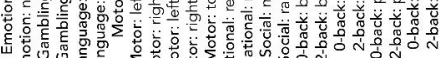

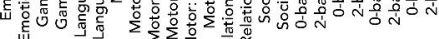

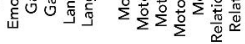

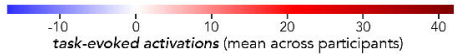

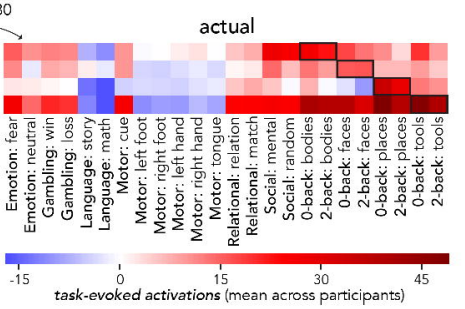


bodies

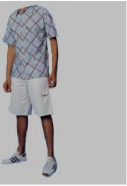

faces

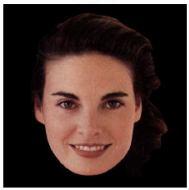

B bodies functional complex

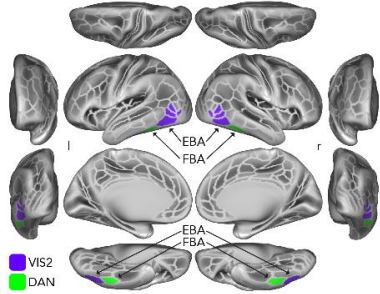

D places functional complex
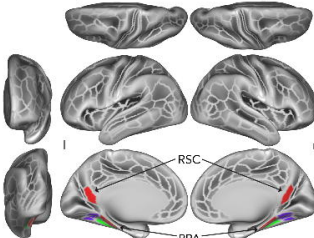

VIS2

DAN
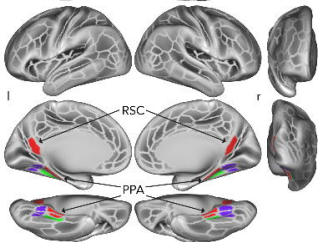

places

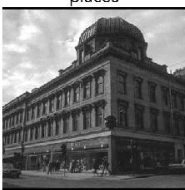

C faces functional complex
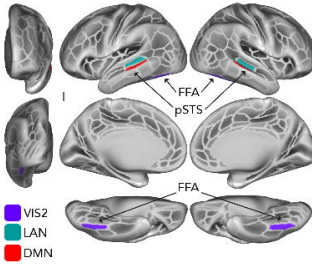

E

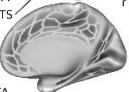

tools functional complex
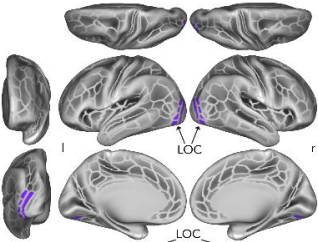

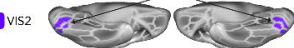

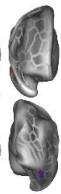

DMN 


\section{A 1}
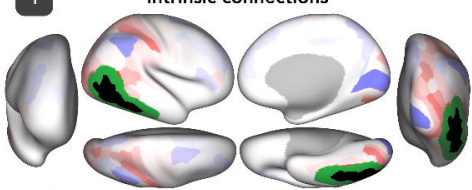

$-0.01$ rsFC estimates (combined FC, seeded to $\mathrm{rEBA} \& \mathrm{FFBA}$ )
4

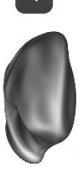

predicted activation to bodies

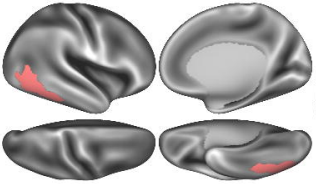

activations ( $\beta$, rEBA \& rFBA predicted)

source vertices excluded from analysis
B

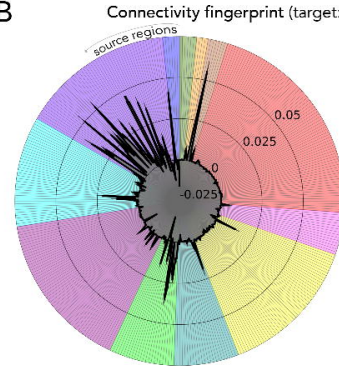

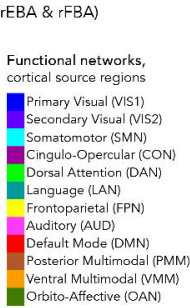

rsFC, $95 \%$ confidence interval
2 actual activations to bodies

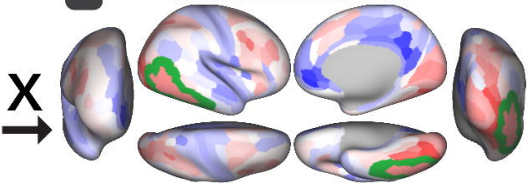

$-20.0$

activations ( $\beta$, rEBA \& rFBA held out)

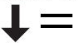

3

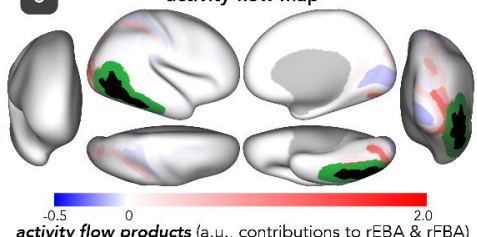

activity flow products (a.u., contributions to rEBA \& rFBA)

to-be-predicted target region (held out)
C

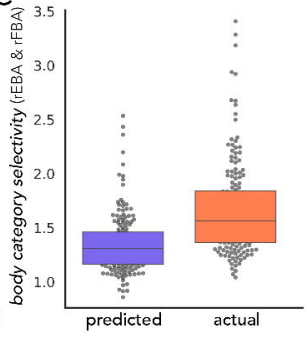

$\mathrm{D}$

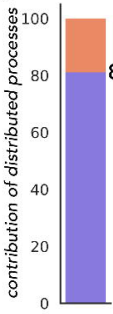

Activity flows for body categories (into: rEBA \& rFBA)

E

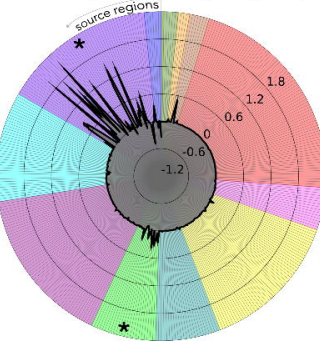

Functional networks cortical source regions

Primary Visual [VI51) Secondary Visual (VIS2) 5omatomotor (SMN) Cingulo-Opercular (CON) Dorsal Attention (DAN) Language (LAN) Frontoparietal (FPN) Auditory (AUD) Default Mode (DMN) Posterior Multimodal (PMM) Ventral Multimodal (VMM) Orbito-Affective (OAN)

- flows, $95 \%$ confidence interval
Network contributions to response profile (predicting: rEBA \& rFBA)

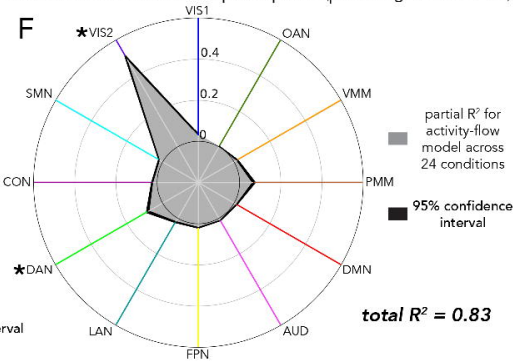



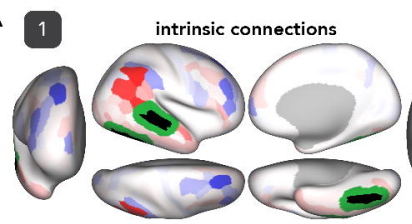

$-0.01$ rsFC estimates (combined FC, seeded to rFFA \& rpSTS)
$1=$
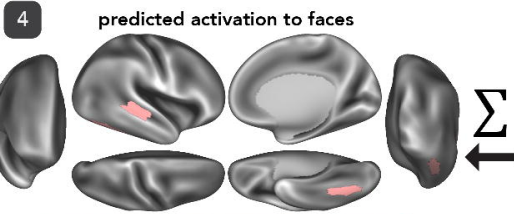

3
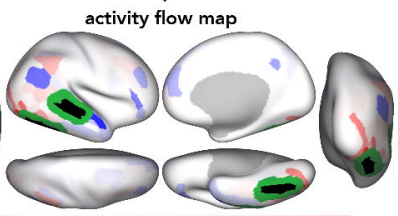

$-0.35$

activity flow products (a.u., contributions to rFFA \& rpSTS)

activations ( $\beta$, rFFA \& IPSTS predicted)

42.0

source vertices excluded from analysis

B
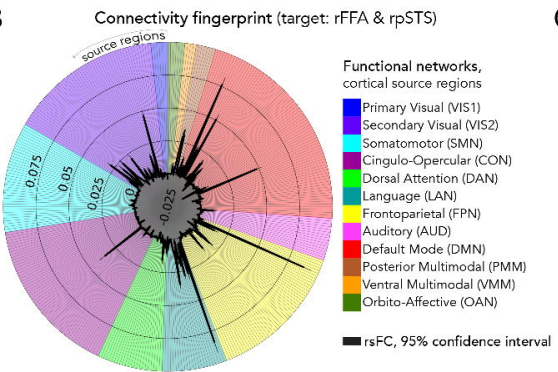

to-be-predicted target region (heid out)
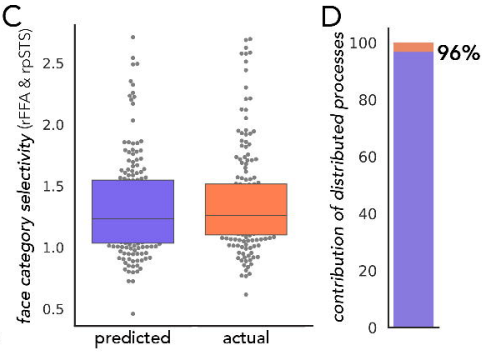

Activity flows for face categories (into: rFFA \& rpSTS)

$E$

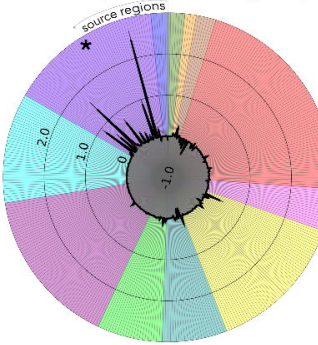

Functional networks, cortical source regions

Primary Visual (VIS1) Secondary Visual (VIS2) Somatomotor (SMN) Cingulo-Opercular (CON) Dorsal Attention (DAN) Language (LAN) Frontoparietal (FPN)

Auditory (AUD) Default Mode (DMN) Posterior Multimodal (PMM) Ventral Multimodal (VMM) Orbito-Affective (OAN)
Network contributions to response profile (predicting: rFFA \& rpSTS)

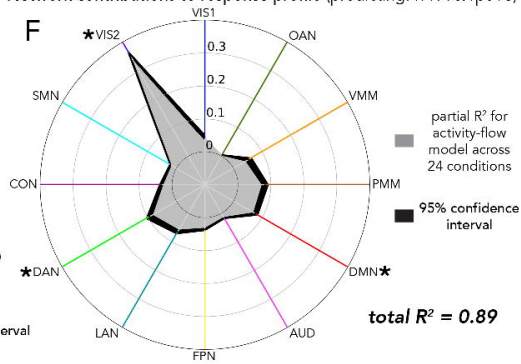


1 intrinsic connections
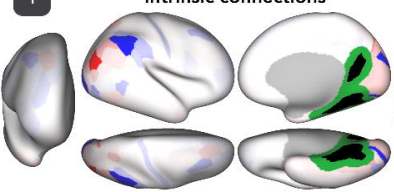

$-0.01$

rsFC estimates (combined FC, seeded to $P$ PPA \& rRSC)

4

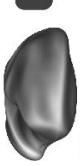

0

activations ( $\beta$, rPPA \& rRSC predicted)

source vertices excluded from analysis

Connectivity fingerprint (target: rPPA \& rRSC)

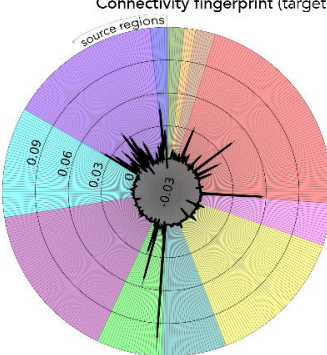

Functional networks, cortical source regions

Primary Visual (VIS1)

Secondary Visual (VIS2)

Somatomotor (SMN)

Cingulo-Opercular (CON)

Dorsal Attention (DAN)

Language (LAN)

Frontoparietal (FPN)

Auditory (AUD)

Default Mode (DMN)

Posterior Multimodal (PMM)

Ventral Multimodal (VMM)

Orbito-Affective (OAN)

- rsFC, 95\% confidence interval
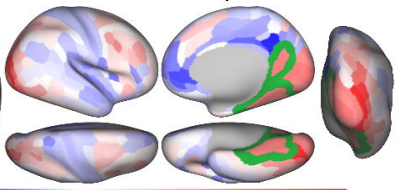

$-21.0$

activations ( $\beta$, rPPA \& rRSC held out)

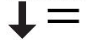

3

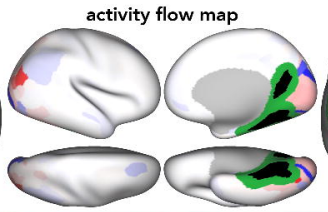

2.0

$-0.3 \quad 0$

activity flow products (a.u., contributions to rPPA \& rRSC) to-be-predicted target region (held out)

C

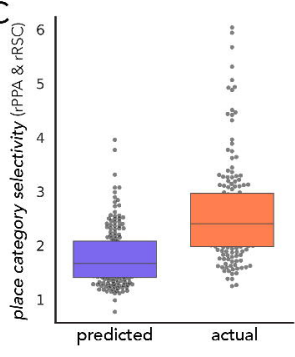

D

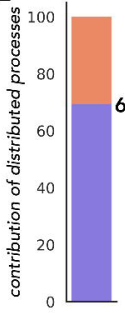

Activity flows for place categories (into: rPPA \& rRSC)

E

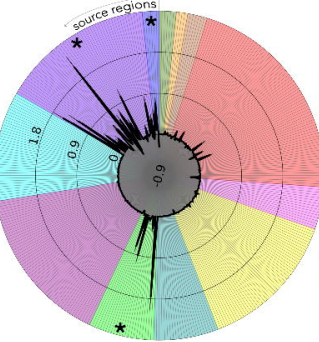

Functional networks,

cortical source regions

Primary Visual (VIS1)

Secondary Visual (VIS2)

Somatomotor (SMN)

Cingulo-Opercular (CON)

Dorsal Attention (DAN)

Language (LAN)

Frontoparietal (FPN)

Auditory (AUD)

Default Mode (DMN)

Posterior Multimodal (PMM)

Ventral Multimodal (VMM)

Orbito-Affective (OAN)

- flows, $95 \%$ confidence interval

Network contributions to response profile (predicting: rPPA \& rRSC)

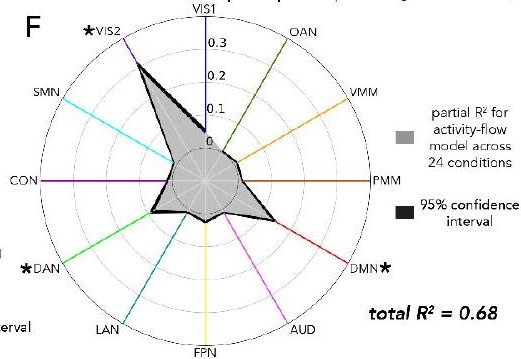


1 intrinsic connections
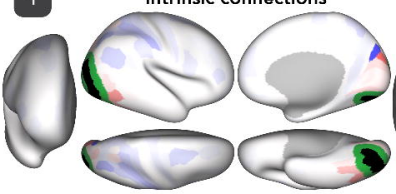

$-0.010$ rsFC estimates (combined $F C$, seeded to $\mathrm{LLOC}$ )
4

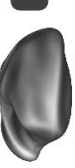

o

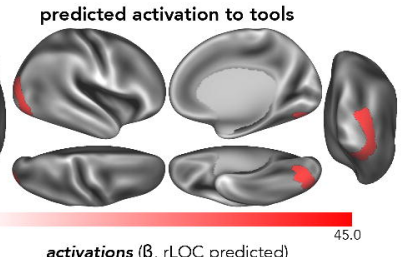

source vertices excluded from analysis

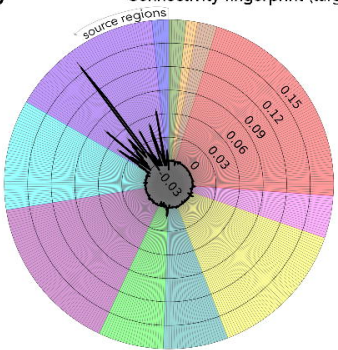

Connectivity fingerprint (target: ILOC)

Functional networks, cortical source regions

Primary Visual (VIS1)

Secondary Visual (VIS2)

Somatomotor (SMN]

Cingulo-Opercular (CON)

Dorsal Attention (DAN)

Language (LAN)

Frontoparietal (FPN)

Auditory (AUD)

Default Mode (DMN)

Posterior Multimodal (PMM)

Ventral Multimodal (VMM)

Orbito-Affective (OAN)

refC, $95 \%$ confidence interval

C

Activity flows for tool categories (into: rLOC)
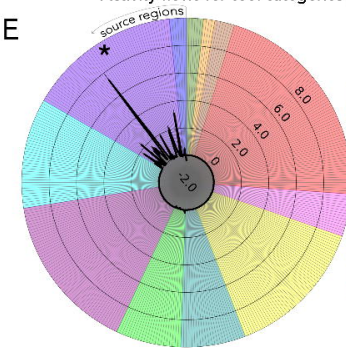

Functional networks, cortical source regions

Primary Visual (V|S1) Secondary Visual (NIS2) Somatomotor (SMN) Cingulo-Opercular (CON) Dorsal Attention (DAN) Language (LAN) Frontoparietal (FPN) Auditory (AUD) Default Mode (DMN) Posterior Multimodal (PMM) Ventral Multimodal (VMM) Orbito-Affective (DAN)

flows, $95 \%$ confidence interval
2 actual activations to tools
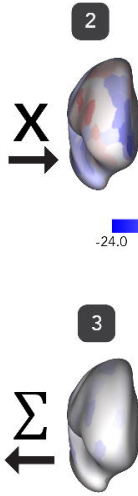

$-0.05 \quad 0$

activity flow products (a.u., contributions to rLOC)

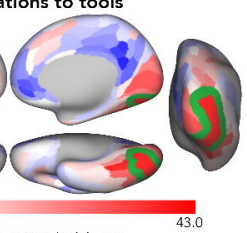

$1=$
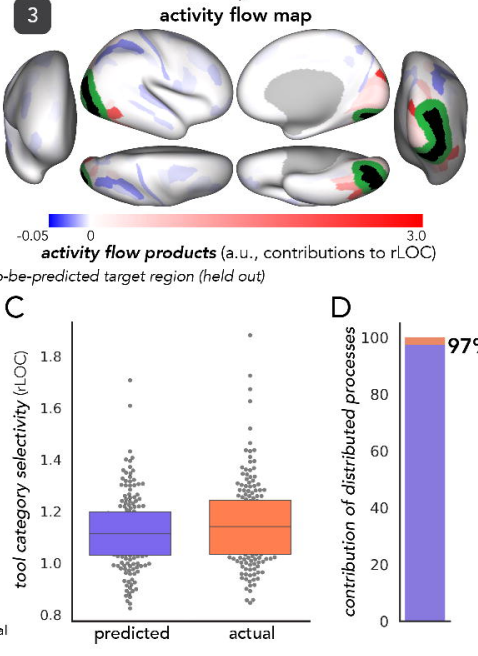

to-be-predicted target region (held out)

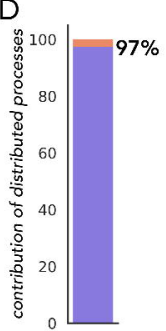

Network contributions to response profile (predicting: $\mathrm{rLOC}$ )

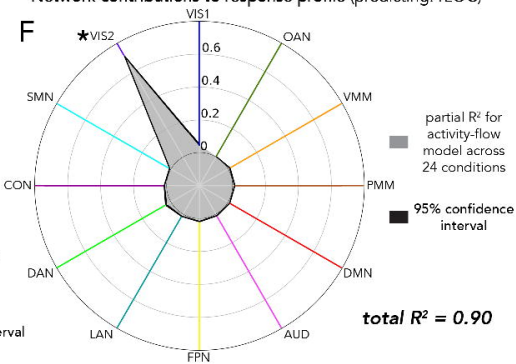




\section{out activity in $j$}

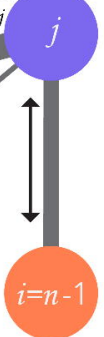

$j$ 's predicted activity $=\sum_{i \neq j}($ 's actual activity $\mathbf{X}$ connectivity $i \rightarrow j$ )

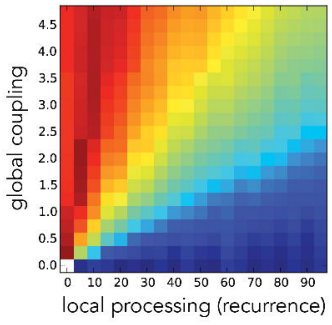

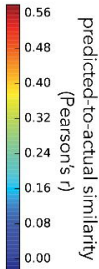

2 actual task activations ( $j$ held out)

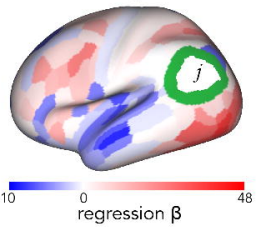

$31=$

4 activity flow map (contributions to $j$ )
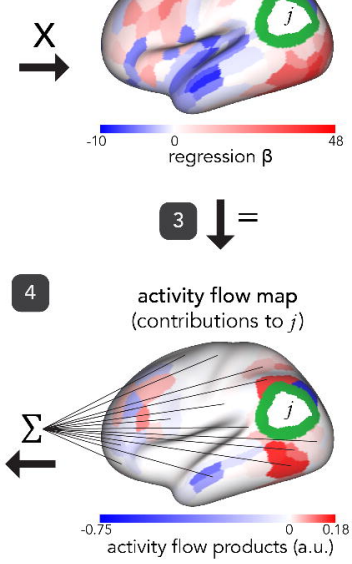

6

prediction accuracy indices $\left(r, R^{2}, \mathrm{MAE}\right)$

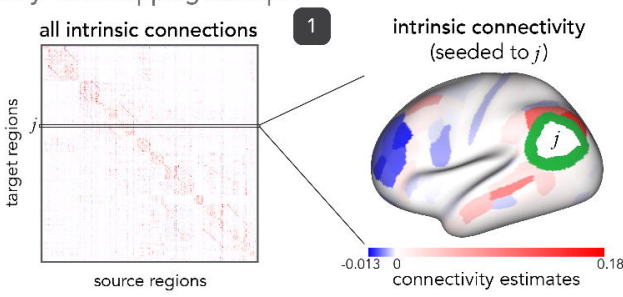

all predicted task activations

all actual task activations

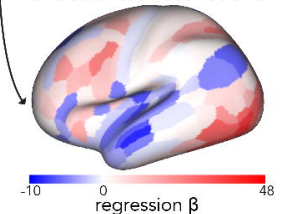

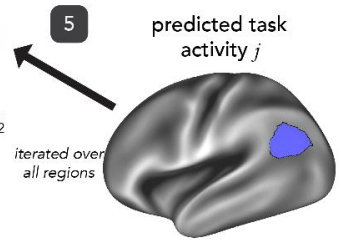

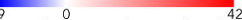
prediction estimates (a.u.)

iterated over all regions excluded from analysis 